\title{
Realizing room-temperature strong coupling of single-exciton with plasmons by controlling quantum exceptional point
}

\section{Wei Li}

State Key Laboratory of Optoelectronic Materials and Technologies, School of Physics, Sun Yat-sen University

\section{Renming Liu}

School of Physics and Electronics, Henan University

Junyu Li

State Key Laboratory of Optoelectronic Materials and Technologies, School of Physics, Sun Yat-sen University

\section{Jie Zhong}

State Key Laboratory of Optoelectronic Materials and Technologies, School of Physics, Sun Yat-sen University

\section{Huanjun Chen}

Guangdong Province Key Laboratory of Display Material and Technology, Sun Yat-sen University https://orcid.org/0000-0003-4699-009X

\section{Xue-Hua Wang ( $\square$ wangxueh@mail.sysu.edu.cn)}

State Key Laboratory of Optoelectronic Materials and Technologies, School of Physics, Sun Yat-sen University https://orcid.org/0000-0003-1324-1471

\section{Article}

Keywords: single-exciton, plasmon, room-temperature, quantum exceptional point, strong coupling

Posted Date: March 1st, 2021

DOl: https://doi.org/10.21203/rs.3.rs-228136/v1

License: (c) (1) This work is licensed under a Creative Commons Attribution 4.0 International License. Read Full License 


\section{Realizing room-temperature strong coupling of single-exciton with}

\section{plasmons by controlling quantum exceptional point}

Wei Li, ${ }^{1,4}$ Renming Liu, ${ }^{1,2,4}$ Junyu Li, ${ }^{1}$ Jie Zhong, ${ }^{1}$ Huanjun Chen ${ }^{3}$, and Xue-Hua Wang ${ }^{1, *}$

${ }^{1}$ State Key Laboratory of Optoelectronic Materials and Technologies, School of Physics, Sun Yatsen University, Guangzhou 510275, China

${ }^{2}$ Institute of Photobiophysics, School of Physics and Electronics, Henan University, Kaifeng 475004, China

${ }^{3}$ School of Electronics and Information Technology, Sun Yat-sen University, Guangzhou 510006, China

${ }^{4}$ These authors contributed equally: Wei Li, Renming Liu.

*Correspondence to: wangxueh@mail.sysu.edu.cn.

Single-exciton strong coupling with plasmons is highly desirable for exploiting room-temperature quantum devices and applications. However, the large plasmon decay makes the realization of such strong coupling extremely difficult. To overcome this challenge, here we propose an effective approach to easily achieve the single-exciton strong coupling at room temperature by controlling quantum exceptional point (QEP) of the coupling system via matching the decay between the localized plasmon mode (LPM) and exciton. The good match can be reached by suppressing the LPM's decay with the use of a leaky Fabry-Perot cavity. Experimental results show that the LPM's decay linewidth is greatly compressed from $\sim 45 \mathrm{~nm}$ to $\sim 15 \mathrm{~nm}$, which is close to the excitonic linewidth $(\sim 10 \mathrm{~nm})$, pushing their interaction from the Fano interference into the strong coupling. Our work opens a new way to flexibly control the QEP and more easily realize the single-exciton strong coupling in ambient conditions. 
Strong light-matter interactions has been a central subject in quantum optics and nano optics, and has exhibited a wealth of applications in quantum technologies, such as quantum logic gates ${ }^{1}$, single-atom lasers ${ }^{2}$ and quantum information processing ${ }^{3-6}$, which rely on a single quantum emitter (QE) strongly interacting with a single-mode field via the coherent exchange of energy between the matter and light components ${ }^{7,8}$. Under this condition, light and matter give up their separate identities and new dressed states arise, which modulate the optical response of the system to external stimuli depending on the coupling strength ${ }^{9}$. To achieve the single-QE strong interactions,

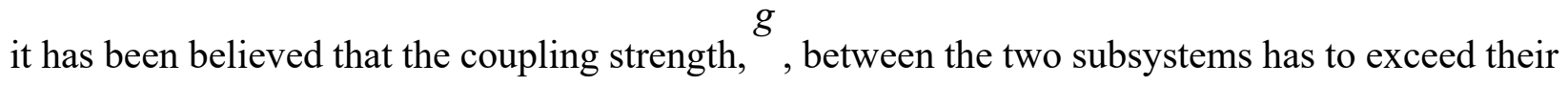
dissipative decays ${ }^{10-12}$.

Generally, there are two ways to realize the single-QE strong coupling with photonic modes. One way is to make the dissipative decays of the two subsystems very small, such as the traditional atomic $^{13,14}$ and solid-state microcavity systems ${ }^{7,15-17}$ with ultrahigh quality factors (i.e., ultralow photon loss) operated at strict cryogenic temperature and in ultrahigh vacuum that remarkably reduces the dissipative decay of the QE. The other is to greatly enhance the coupling strength $g$ for the room-temperature quantum strong coupling, such as plasmonic systems coupled with the QEs. In the past two decades, this emerging field of the room-temperature quantum strong coupling has made great progresses by squeezing the mode volume $\left(V_{\mathrm{m}}\right)$ of the localized plasmon modes (LPMs) to greatly improve the coupling strength ${ }^{18-28}$. Although recent experiments have successfully demonstrated the room-temperature strong coupling of a few QEs and the LPMs with ultrasmall $V_{\mathrm{m}}{ }^{26-29}$, realizing the deterministic strong coupling of a single-QE with the LPMs at ambient conditions remains a great challenge, because it is almost impossible to achieve a further significant reduction of the mode volume.

In this article, we propose a novel strategy to make it easier to realize the single-exciton strong 
coupling with the LPM at room temperature by lowering the critical coupling-strength (or coupling-energy) at the quantum exceptional point (QEP). The QEP is the singularity in Hilbert space of the quantum coupled system described by non-Hermitian Hamiltonians, where the eigenenergies and corresponding eigenstates simultaneously coalesce. The research on the QEPs and associated applications is becoming an emerging field ${ }^{30-33}$. The eigenenergies of a nonHermitian quantum system consisting of a two-level single exciton coupled with an optical mode (cavity mode or LPM) can be written as $8,28,34,35$

$$
\varepsilon_{ \pm}^{l}=\left(\varepsilon_{c}+\varepsilon_{d}\right) / 2-i\left(\gamma_{d}+\gamma_{c}\right) / 4 \pm \Delta_{l s}, \text { with } \Delta_{l s}=\sqrt{g^{2}+\left[\delta+i\left(\gamma_{d}-\gamma_{c}\right)\right]^{2} / 16}
$$

where $2 \Delta_{l s}$ is the level splitting of the coupled system, $g$ is the coupling strength (i.e. coupling energy) between the single exciton and optical mode; $\delta=\varepsilon_{d}-\varepsilon_{c}$ is the detuning between the levels of the optical mode $\left(\varepsilon_{d}\right)$ and the exciton $\left(\varepsilon_{c}\right) ; \gamma_{d}$ and $\gamma_{c}$ are the decay linewidths of the corresponding levels, respectively. At resonance $\delta=0$, the critical coupling-strength at the QEP with $\Delta_{l s}=0$ is

$$
g_{Q E P}=\left|\gamma_{d}-\gamma_{c}\right| / 4
$$

As shown in Supplementary Fig. 1a, when $g<g_{Q E P}$, the system is in the weak coupling regime, in which the level (real part) is degeneracy but with two decay channels (corresponding to the two images of the eigenenergies); when $g>g_{Q E P}$, the system is in the strong coupling regime, in which the level (real part) splits but with only one decay channel (the image part of the eigenenergies is degeneracy). Therefore, the larger the critical coupling-strength $g_{Q E P}$ of the QEP is, the more difficult the realization of the strong coupling is. In other words, if $\gamma_{d}$ and $\gamma_{c}$ are approximate match, the observation of the level splitting characterizing the strong coupling becomes much easier (Supplementary Fig. 1b). 
In traditional atomic and solid state systems operated at cryogenic temperature and in ultrahigh vacuum, the dissipative decays of the coupling systems are very small (for instance, $\gamma_{d} \approx 0.197$ $\mathrm{nm}=42.3 \mathrm{GHz}$ and $\left.\gamma_{c} \approx 0.1 \mathrm{~nm}=21.5 \mathrm{GHz}\right)^{35}$, which makes the critical $g_{Q E P}$ at the QEP is very low, and then an extremely small coupling strength (such as $g \approx 0.096 \mathrm{~nm}=20.6 \mathrm{GHz})^{35} \mathrm{can}$ satisfy the strong coupling condition well. However, the decay linewidths $\gamma_{d}$ and $\gamma_{c}$ in the hybrid system consisting of a single exciton coupled with a LPM at room temperature are more than two orders of magnitude larger than those in the traditional atomic and solid state systems operated at cryogenic temperature, and $\gamma_{d}$ is much larger than the $\gamma_{c}$. These typical characteristics lead to a huge critical $g_{Q E P}$ at the QEP and make the single-exciton strong coupling extremely difficult. From Eq. (2), if the $\gamma_{d}$ can be suppressed to well match with $\gamma_{c}$, the critical $g_{Q E P}$ will be greatly reduced even down to zero, which makes the realization of singleexciton strong coupling very easy and the strict conditions such as cryogenic temperature and ultrahigh vacuum become unnecessary.

\section{Results}

Pseudo-strong coupling between single-exciton and plasmon at room temperature. To demonstrate the challenge in realizing such single-exciton strong coupling at room temperature, we firstly fabricated the coupled systems of a single exciton with the LPM by attaching a 1-nmthick TDBC J-aggregate layer (treated with 1- $\mu \mathrm{M}$ dye solution, see Supplementary Fig. 2) on individual $\mathrm{Au}$ (core)-Ag (shell) nanorods (Au@Ag NRs) with the electrostatic self-assembly method (see Methods for details) ${ }^{28}$. This approach enables single J-aggregate excitons be successfully assembled on the sharp corners of the cuboid $\mathrm{Au} @ \mathrm{Ag} \mathrm{NR}$, and ensures the interaction distance between the exciton and the plasmonic mode to be within $1.0 \mathrm{~nm}^{28}$. This ultrashort 
interaction distance provides advantages to realize strong coupling at the single-QE level.

Experimentally, the dark-field scattering measurements of individual Au@Ag NR/J-aggregates hybrids on the indium tin oxide (ITO)-coated glass substrate are firstly performed. Figure 1a shows the scattering spectra of the bare cuboid Au@Ag NR with the size of $65 \times 36 \mathrm{~nm}$, which is on resonance with the exciton absorption. The measured scattering spectrum from the hybrid system consisting of an $\mathrm{Au} @ \mathrm{Ag}$ NR covered around with single-layer J-aggregates is plotted by the solid pink curve with an evident spectral splitting of $\mathrm{h} \Omega_{R} \sim 69 \mathrm{meV}$ (Fig. 1b). The theoretical scattering spectrum is displayed by the dotted black curve, which is obtained by the following Eq. (3) (detailed description in Supplementary Note 2$)^{28,36}$ with $N=1, \gamma_{d} \sim 159 \mathrm{meV}$ and $\gamma_{c} \sim 36 \mathrm{meV}$, and the calculated $g \sim 29.7 \mathrm{meV}$ at the average interaction distance of $0.5 \mathrm{~nm}$ between the exciton and the cuboid Au@Ag NR.

$$
\sigma(\omega) \propto-\operatorname{Im}\left\{\mathrm{h} \omega-\varepsilon_{d}+i \frac{\gamma_{d}}{2}-\frac{g^{2}}{\mathrm{~h} \omega-\varepsilon_{c}+i \frac{\gamma_{c}}{2}}\right\}^{-1}
$$

Here, $N$ is the number of the excitons coherently coupled to a plasmon mode. $g$ is calculated as a mean coupling strength $\bar{g}$ using Eq. (S4) (Supplementary Note 2) based on a normalized electric field (EF) $f_{d}(r)=0.189$ (Supplementary Note 3), $V_{m} \sim 91 \mathrm{~nm}^{3}$ (Supplementary Note 4) and the transition dipole moment $\mu_{c} \sim 0.44 \mathrm{e} \mathrm{nm}$ for a single J-aggregate exciton (Supplementary Note 5). The calculation details of $g$ can be found in Supplementary Note 6 and Supplementary Table 1. The excellent agreement between the experimental and theoretical results in Fig. 1b demonstrates that we have successfully fabricated the coupled system of a single exciton with the LPM by treating the samples with the $1-\mu \mathrm{M}$ dye solution, and the coupling strength $g$ between the single exciton and LPM is about $29.7 \mathrm{meV}$. It is worth emphasizing that in our calculations 
with Eq. (3), $N$ is the only fitting parameter to be determined.

In order to further validate the above-mentioned conclusions, we exam the dispersion of spectral splitting $\mathrm{h} \Omega_{R}$ with different detunings for individual $\mathrm{Au} @ \mathrm{Ag} \mathrm{NR} / \mathrm{J}$-aggregate hybrids with different NR size. The normalized scattering spectra of individual Au@Ag NR/J-aggregate hybrids with different detunings are displayed in Fig. 1c, in which a vague anticrossing behavior with $\mathrm{h} \Omega_{R} \sim 69 \mathrm{meV}$ can be observed. The scattering spectra of three typical single Au@Ag NR/Jaggregate hybrids with different detunings can be found in Supplementary Fig. 7. Figure 1d gives the theoretical dispersion on the detuning ( $\delta=\varepsilon_{d}-\varepsilon_{c}$ ) obtained by Eq. (3) with $N=1, g \sim 29.7$ $\mathrm{meV}, \varepsilon_{c}=2.11 \mathrm{eV}, \gamma_{c}=36 \mathrm{meV}$ and $\bar{\gamma}_{d}=158 \mathrm{meV}$ (Supplementary Fig. 8a), in which $\varepsilon_{d}$ is calculated according to the particle sizes of individual Au@Ag NRs. Once again, we observe the reasonably good agreement between experimental and theoretical results.

It is more clearly demonstrated by giving the quantum step statistics for identifying different $N$ in Fig. 1e that most of the individual Au@Ag NR/J-aggregates hybrids prepared with the 1- $\mu \mathrm{M}$ dye solution are indeed the coupled systems of a single exciton interacting with the NR. For convenience, we call such individual hybrids as single-exciton-coupled NRs. The contrastive experiment and theory results for the hybrids isolated from the sample treated with the $3-\mu \mathrm{M}$ dye solution are exhibited in Supplementary Figs. 9 and 10, which illustrate that most of the individual hybrids are the coupled systems of multiple (three or four) excitons with the LPM (Fig. 1f). 

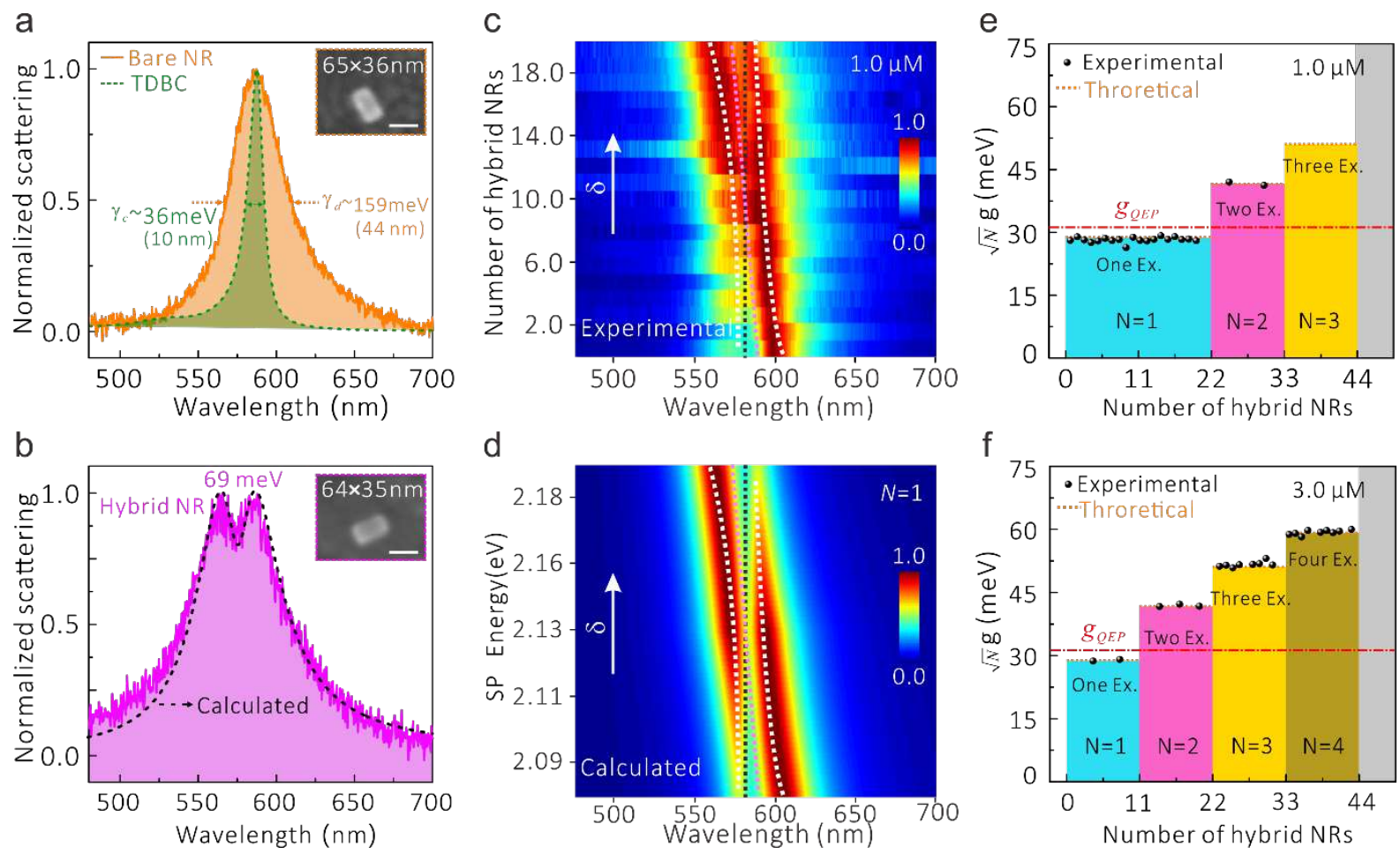

Figure 1 | LPM-exciton couplings in single Au@Ag NR/J-aggregate hybrids on ITO substrate.

a Normalized scattering of a bare Au@Ag NR resonant to the exciton absorption (dashed green curve) with $\varepsilon_{c}=2.11 \mathrm{meV}$. b Normalized scattering spectra of a strongly coupled Au@Ag NR/Jaggregate hybrid at resonance condition. The dotted black line is the theoretical result calculated using Eq. (3) with $N=1$. c Normalized scattering spectra of the individual Au@Ag NR/Jaggregate hybrids isolated from the ensembles treated with 1.0- $\mu$ Mdye solution, and ordered according to the detuning. d Extinction spectra calculated using Eq. (3) with $N=1$ for the same samples as in (c). e, f Quantum step statistics of the effective coupling strength $(\sqrt{N} g)$ extracted (using the method proposed in ref. ${ }^{37}$.) from the measured individual hybrids isolated from the ensembles treated with (e) $1.0-\mu \mathrm{M}$ and (f) $3.0-\mu \mathrm{M}$ dye solution, respectively. The insets in (a), (b) are SEM images of the measured nanostructures, and the scale bar is $50 \mathrm{~nm}$.

It is very surprising to notice that the coupling strength $g \sim 29.7 \mathrm{meV}$ between the single- 
exciton and LPM is less than the critical coupling-strength, $g_{Q E P}=\left|\gamma_{d}-\gamma_{c}\right| / 4=30.75 \mathrm{meV}$, at the QEP, indicating that the level splitting of the coupled system has not occurred. It implies that these hybrid samples of a single-exciton coupled with plasmonic NRs are in the weak coupling regime, despite of the fact that the spectral splitting is evident. This interesting phenomenon has been detailedly discussed in our recent theory work and the corresponding interaction is assigned to the pseudo-strong coupling regime ${ }^{37}$, in which the spectral splitting arises from the Fano resonance ${ }^{22,29,38}$. This result also gives an experimental proof to the prediction made in ref. ${ }^{37}$ that the equality assumption of spectral splitting and level splitting is invalid in usual plasmon-exciton coupling systems with large dissipative decays and remarkable decay mismatch between the two coupling subsystems. In the following text, we will theoretically and experimentally demonstrate how the proposed approach, that lowers the critical coupling-strength at the QEP by suppressing the LPM's decay $\gamma_{d}$ to match with the exciton's decay $\gamma_{c}$, and pushes the interaction of the single exciton and LPM from the Fano resonance into strong coupling.

Theoretical design a leaky Fabry-Perot (FP) cavity to suppress the plasmon decay. It is known that the plasmon modes can be engineered by the electromagnetic (EM) environment around metal nanostructures to form hybrid plasmonic-photonic modes with small $V_{m}$ and moderate $Q^{39-41}$. Such hybrid modes have potential applications in emission enhancement ${ }^{42,43}$, second-harmonic resonances $^{44}$ and molecular or nanoparticle (NP) detections ${ }^{45-47}$. Here, we reveal that the dissipative decay $\gamma_{d}$ of the LPMs supported by metal NPs can be significantly suppressed by a leaky FP cavity to match well with the excitonic decay linewidth $\gamma_{c}$. This strategy is a general approach for a variety of metal nanostructures. Without loss of generality, we first use a most common plasmonic nanostructure of $\mathrm{Au} \mathrm{NR}$ to show this general strategy. Figure 2a shows the 
schematic diagram of such a plasmonic-photonic hybrid system by loading a single Au NR on the surface of a leaky FP cavity constructed by three dielectric layers of $\mathrm{Si} / \mathrm{SiO}_{2} / \mathrm{Si}_{3} \mathrm{~N}_{4}$. The dashed gray curve in Fig. 2b gives some typical FP-cavity modes in the spectral region of 550-775 nm. The EFs of the FP-cavity modes can be leaked into the free space and formed an EM environment (Fig. 2c) above the up surface of the cavity. When the metal nanoparticles are loaded in this EM environment, their LPMs can be significantly tailored by these leaky FP-cavity modes. The solid color curves in Fig. 2b shows the light scattering of the Au NRs with different aspect ratios loaded on the surface of the leaky FP cavity, demonstrating significant sculption of their LPMs by these FP-cavity modes. New engineered-LPMs (ELPMs) near the FP-cavity modes (channels) of $\mathrm{TM}_{1} \sim$ $631 \mathrm{~nm}, \mathrm{TM}_{2} \sim 664 \mathrm{~nm}$ and $\mathrm{TM}_{3} \sim 700 \mathrm{~nm}$ occurs when the different LPMs of three Au NRs with different sizes are respectively resonant with the three FP-cavity modes. More interestingly, the decay linewidths of these three ELPMs are $\sim 11.8,13.5$ and $16.4 \mathrm{~nm}$, which are approximately compressed to $\sim 1 / 3$ of those $(39.7,41.3$, and $43.6 \mathrm{~nm})$ of the original LPMs on the bulk $\mathrm{Si}_{3} \mathrm{~N}_{4}$ substrate.

When the synergistic interaction between the NR and the FP-cavity mode TM2 is on resonance, interestingly, the EF intensity on the surface of the NR can be improved compared to the two separate entities without synergistic interactions (Fig. 2d). Figure 2e shows that the total EF $\left(|E|^{2}\right)$ on the surface of the FP-cavity-engineered NR, which has been enhanced by $\sim 3$ times compared to the NR located on the bulk $\mathrm{Si}_{3} \mathrm{~N}_{4}$ substrate. The merits of the lowered dissipative decays ${ }^{48,49}$ and the enhanced plasmonic EFs make the cavity-engineered NRs an ideal platform for implementing the quantum manipulation of light-matter interactions at room temperature. Next, we experimentally demonstrate the suppression of the dissipative decay for the Au@Ag NRs by applying this general strategy. 


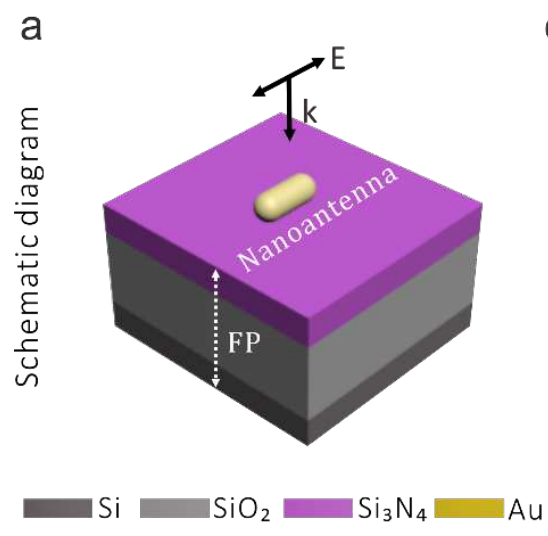

b

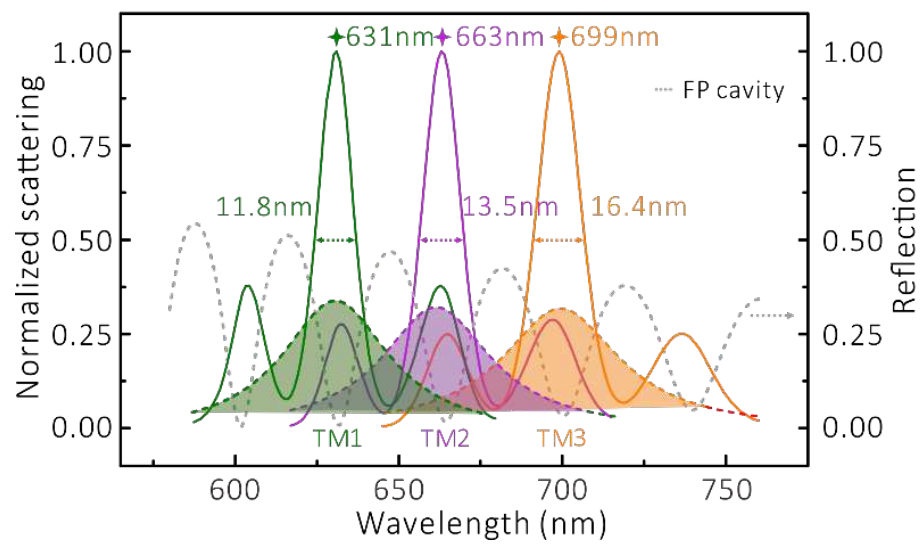

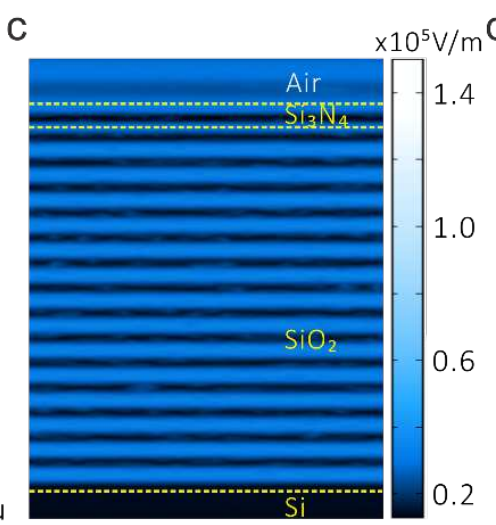

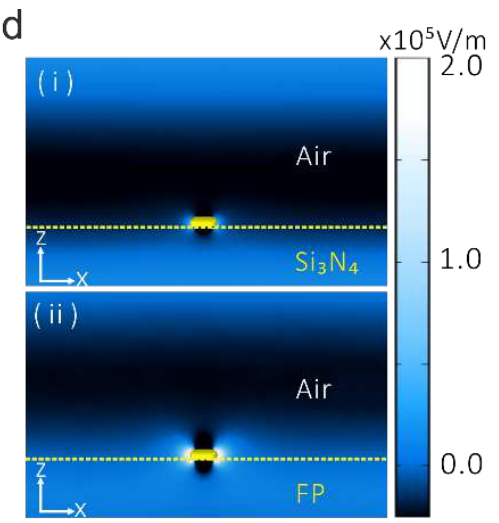

e

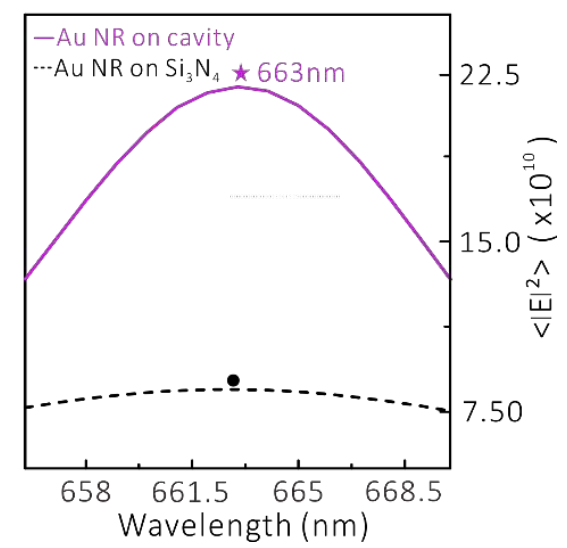

Figure 2 | Synergistic nanoantenna-microcavity hybrid for plasmonic decay suppression and

local EF enhancement. a Schematic diagram of the synergistic Au NR/FP-cavity hybrid system.

b Normalized scattering calculated for the Au NRs with different aspect ratios localized on the surface of the leaky FP cavity. The solid and dashed color curve represents the normalized scattering of the $\mathrm{Au}$ NRs on the FP cavity and on the bulk $\mathrm{Si}_{3} \mathrm{~N}_{4}$ substrate, respectively. The dashed gray curve is the reflectivity of the bare FP cavity. Here, the length $l$ and radius $r$ of the Au NR are 45.2, 54.0, 61.8 and $12 \mathrm{~nm}$, respectively; c Simulated total EF through the middle of the bare FP cavity. d Simulated $x$-component of the total EF, $E_{x}$, through the middle of the Au NR ( $l=54$ nm) on the bulk $\mathrm{Si}_{3} \mathrm{~N}_{4}$ substrate (i) and the FP cavity (ii), respectively. e Average near-field intensity $\left\langle|E|^{2}\right\rangle$ on the surface of the Au NR $(l=54 \mathrm{~nm})$ as functions of wavelength for the NR 
located on the bulk $\mathrm{Si}_{3} \mathrm{~N}_{4}$ substrate and the FP cavity, respectively. In simulations, $t_{S_{3} N_{4}}=200 \mathrm{~nm}$, $t_{\mathrm{SiO}_{2}}=4035 \mathrm{~nm}$, silica is the substrate.

Experimental realization of the plasmonic decay reduction. Figure 3 a depicts the schematic of the dark-filed scattering measurements for the Au@Ag NR located on the surface of the leaked FP cavity. The reflection spectrum (dashed gray curve) of the leaky FP cavity with $t_{\mathrm{SiO}_{2}}=3008 \mathrm{~nm}$ can be found in Fig. 3b, showing three discrete cavity-modes (TM1', TM2' and TM3') in the spectral region of 575-700 $\mathrm{nm}$. The experimental result agrees well with the theoretical one (solid cyan curve) calculated using the finite element method based on the structural parameters extracted from the measurements. Figure 3c gives the dark-field scattering spectra of three cavity-engineered $\mathrm{Au} @ \mathrm{Ag}$ NRs with aspect ratios of $\sim 1.83,2.12$ and 2.23. It is found that the light scattering of the three Au@Ag NRs reaches their maxima at the FP-cavity modes of TM1' 588, TM2' 627 and TM3' $670 \mathrm{~nm}$, respectively. Just as what we have predicted in Fig. 2b, when the LPM of the bare NR is resonant with a FP-cavity mode, the light scattering will be significantly tailored and largely enhanced by this mode. Since different FP-cavity modes possess different decay linewidths, the three new ELPMs at $\sim 589, \sim 627$ and $\sim 668 \mathrm{~nm}$ have different decay linewidths of $\sim 14.0,18.2$ and $23.0 \mathrm{~nm}$, respectively, which are determined by the bandwidths of the three corresponding FPcavity modes. Compared to the LPMs supported by the bare Au@Ag NRs (Fig. 3d) with similar sizes to those (the inserts) shown in Fig. 3c, the decay linewidths of these ELPMs are respectively compressed to $\sim 1 / 3$ of their original LPMs. Especially, the decay linewidth of $\sim 14.0 \mathrm{~nm}$ for the new ELPM at $\sim 589 \mathrm{~nm}$ matches well with that $\left(\gamma_{c}=10 \mathrm{~nm}\right)$ of the J-aggregate exciton, providing possibilities to largely lower the critical $g_{Q E P}$ at the QEP of the single-exciton-coupled NRs. From Fig. 3b-d we also seen that there are excellent agreements between the measurements and 
the simulated results calculated based on the structural parameters extracted from the corresponding measurements.

On the other hand, for a given FP-cavity mode (for instance, TM1'), after the LPMs of different cuboid NRs sculpted by this mode, the new formed ELPMs have almost same resonance frequencies and decay linewidths with small deviations (Supplementary Fig. 11). This deviation can also be tuned in a broad spectral region by FP cavities with different cavity length $t_{\mathrm{SiO}_{2}}$ (Supplementary Fig. 12). These resonance frequency deviations of the new ELPMs are important for experimental manipulation of light-matter interactions with fine detuning. Figure 3e (i) shows the statistics of the decay linewidths of $14.4 \pm 2.3,18.5 \pm 3.4$ and $23.3 \pm 2.9 \mathrm{~nm}$ for the ELPMs of different nanorods near the TM1', TM2' and TM3', respectively. Compared to the statistical linewidths $(44.8 \pm 4.7,48.3 \pm 6.2$ and $51.4 \pm 5.0 \mathrm{~nm}$ ) of the LPMs for the bare NRs (gray histograms in Fig. 3e (ii)), the decay linewidths of the ELPMs are remarkably narrowed. 

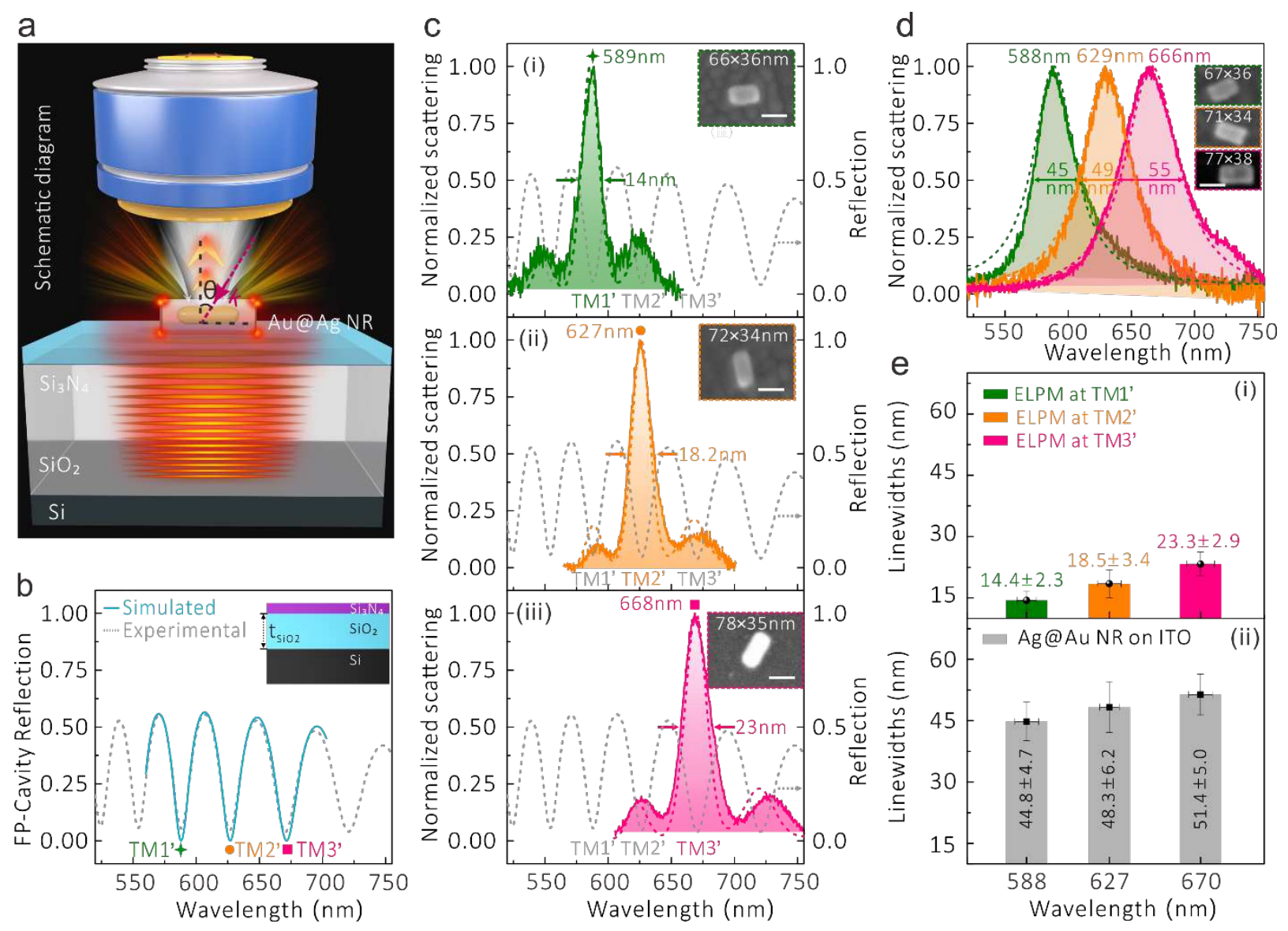

Figure 3 | Scattering measurements of the bare Au@Ag NRs in the EM environments engineered by the leaky FP cavity. a Schematic diagram of dark-field scattering measurement for a single cuboid Au@Ag NR on a leaky FP cavity. b Simulated (solid cyan curve) and measured (dashed grey line) reflectance for the leaky FP cavity with $t_{S_{3} N_{4}}=216.9 \mathrm{~nm}$ and $t_{\mathrm{SiO}_{2}}=3008 \mathrm{~nm}$. The silicon is set as a semi-infinite substrate. c, $\mathbf{d}$ Normalized scattering (solid color curves) for $\mathrm{Au} @ \mathrm{Ag}$ NRs located on (c) the FP cavity (with $t_{\mathrm{Si}_{3} \mathrm{~N}_{4}}=216.9 \mathrm{~nm}$ and $t_{\mathrm{SiO}_{2}}=3008 \mathrm{~nm}$ ) and (d) the ITO-coated glass substrate, respectively. Dashed color curves are corresponding theoretical results calculated using the structural parameters extracted from the measurements. The dashed gray curves represent the experimental reflectance of bare FP cavity. The inserts are corresponding SEM images for the measured cuboid Au@Ag NRs, the scale bar is $50 \mathrm{~nm}$. e Statistics of decay 
linewidths for (i) the ELPMs for the NRs on the FP cavity and (ii) bare LPMs for the NRs on the ITO substrate, note that the statistical wavelength range is $\mathrm{TM}^{\prime}{ }^{\prime} \pm 8, \mathrm{TM}^{\prime} \pm 8$ and $\mathrm{TM}^{\prime}{ }^{\prime} \pm 8 \mathrm{~nm}$, respectively.

Realizing single-exciton strong coupling at room temperature easy by lowering $g_{Q E P}$. We now demonstrate the realization of single-exciton strong coupling in such cavity-engineered $\mathrm{Au} @$ Ag NRs. We placed individual single-exciton-coupled NRs on the top surface of the FP cavity. The measured scattering spectrum is displayed by the black solid line in Fig. 4a, and a clear spectral splitting of $\mathrm{h} \Omega_{R} \sim 61 \mathrm{meV}$ is observed. The dashed pink line is the theoretical scattering spectrum calculated by Eq. (3) with $N=1, g=28.8 \mathrm{meV}$ with an ultrasmall $V_{m} \sim 99 \mathrm{~nm}^{3}$ (Supplementary Notes 4 and 6 and Supplementary Table 1), $\gamma_{c}=36 \mathrm{meV}$ and $\gamma_{d}=54 \mathrm{meV}$ extracted from the experimental measurements in Fig. 4b. It should be mentioned that the aspect ratios of the two different cuboid Au@Ag NRs (Fig. 4a, b) are almost the same, which lead to almost the same resonance wavelengths and decay linewidths (Fig. 3e) when they are locating in the same dielectric environment (Supplementary Table 2). From Fig. 4a, one can see that the experimental and the theoretical results are in a good accordance again. compared to that ( $g_{Q E P}=$ $30.75 \mathrm{meV}$ ) of single-exciton coupling with the Au@Ag NR without cavity-engineered (Fig. 4c), in this case, a dramatically lowered $g_{Q E P}=4.5 \mathrm{meV}$ at the QEP was achieved (Fig. 4d), which makes the level (real part) splitting condition of $g>g_{\text {QEP }}$ to be easily satisfied and the singleexciton strong coupling to be well realized. It should be stressed that this spectral Rabi splitting $\mathrm{h} \Omega_{R} \sim 61 \mathrm{meV}$ (corresponding the coupling strength $g \sim 28.8 \mathrm{meV}$ ) can adequately satisfy the 
other strong-coupling criteria in the previous works, such as $2 g>\gamma_{d}, \gamma_{c}{ }^{24,27,38}$, or $2 g>\sqrt{\left(\gamma_{d}^{2}+\gamma_{c}^{2}\right) / 2}$ or $2 g>\left(\gamma_{d}+\gamma_{c}\right) / 2^{5,8,28,50}$.

Typical dark-field scattering spectra of the single-exciton-coupled NRs with different detuning localized on this leaky FP cavity are presented in Supplementary Fig. 13. More scattering spectra of the single-exciton-coupled NRs ordered according to the detuning shown in Fig. 4e, in which a clear anticrossing behavior with $\mathrm{h} \Omega_{R} \sim 61 \mathrm{meV}$ is observable, agreeing well with the theoretical results calculated using Eq. (3) with $N=1$. Similarly, the quantum steps of the effective coupling strengths, $\sqrt{N} g$, extracted from the measurements are shown in Fig. 4f, from which we see that most of the observed interacting cases are belonging to the single-exciton $(N=1)$ strong coupling $\left(\sqrt{N} g>g_{Q E P}\right)$, a minimum mean number of excitons has been reported so far (Supplementary Table. 3). These results clearly indicate that we have successfully achieved the single-exciton strong coupling at room temperature in single FP-cavity-engineered Au@Ag NRs. 

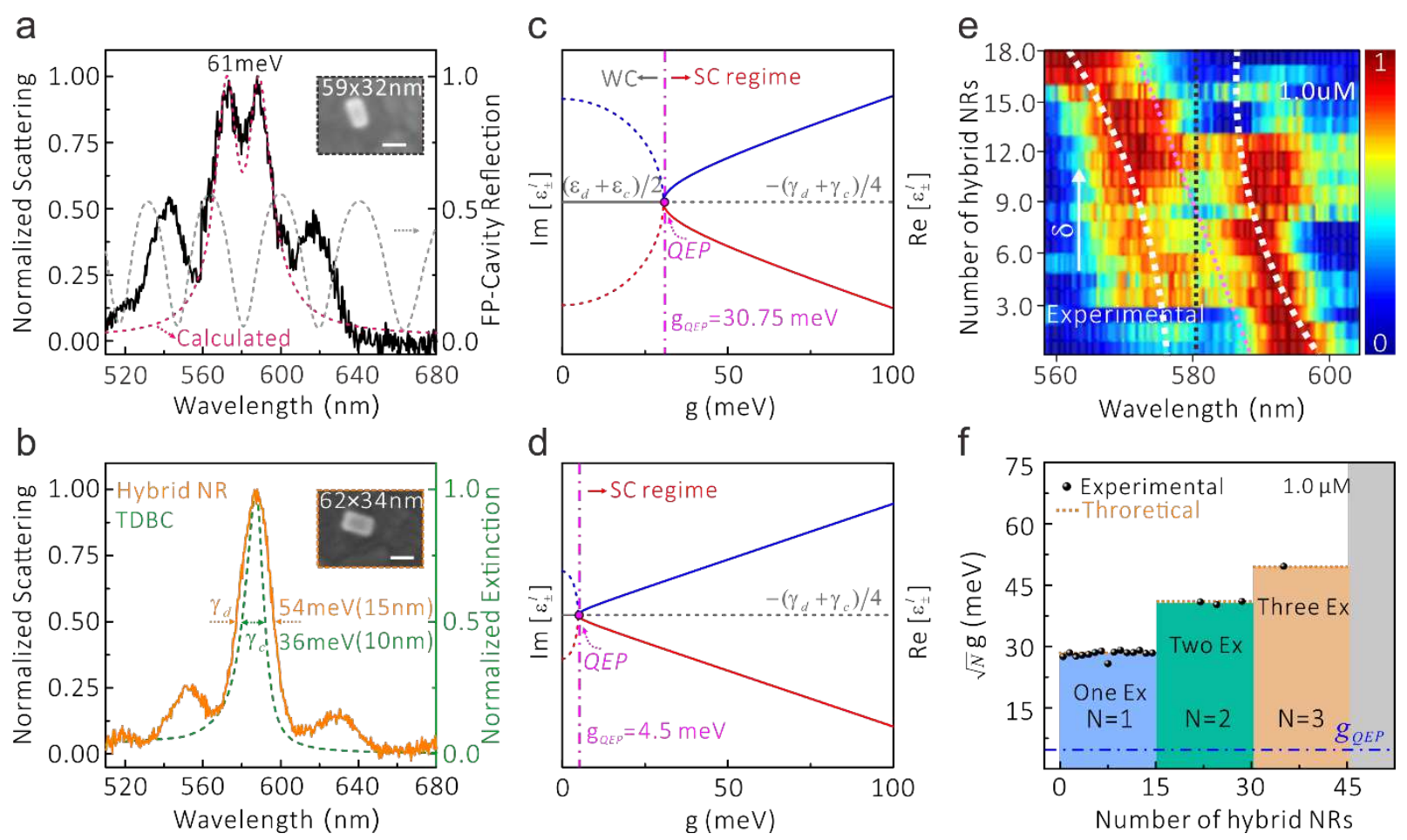

Figure 4 | Single-exciton strong coupling in single-exciton-coupled NRs located on the leaky

FP cavity. a A strongly coupled Au @ Ag NR/J-aggregate hybrid, measured on FP cavity $\left(\mathrm{Si} / \mathrm{SiO}_{2} / \mathrm{Si}_{3} \mathrm{~N}_{4}\right)$. b Extinction spectra of J-aggregate (dashed green curve) solution and Scattering spectra (orange solid curve) of bare Au @ Ag NR with the size of $62 \times 34$ nm located on FP cavity. c, $\mathbf{d}$ Real (solid curve) and imaginary (dashed curve) parts of the eigenenergies in Eq. (1) as a function of $g$ at resonance $\delta=0$ with (c) $\gamma_{d}=159 \mathrm{meV}, \gamma_{c}=36 \mathrm{meV}$; and (d) $\gamma_{d}=54 \mathrm{meV}$, $\gamma_{c}=36 \mathrm{meV}$. WC: Weak coupling; SC: Strong coupling. e Normalized scattering of the individual $\mathrm{Au} @ \mathrm{Ag} \mathrm{NR} / \mathrm{J}$-aggregate hybrids isolated from the sample treated 1.0- $\mu \mathrm{M}$ dye solution, and ordered according to detuning. The dashed white lines are theoretical results calculated using Eq. (3) with $N=1, \bar{\gamma}_{d} \sim 55 \mathrm{meV}$ (Supplementary Fig. $8 \mathrm{~b}$ ), $\gamma_{c} \sim 36 \mathrm{meV}$ and $\bar{g} \sim 28.8 \mathrm{meV}$. f Quantum steps for the effective coupling coefficient, $\sqrt{N} g$, observed as one, two and three-exciton 
coupling cases for the hybrid NRs isolated from the sample treated with the $1.0-\mu \mathrm{M}$ dye solution. The scale bar in the insets of (a) and (b) is $50 \mathrm{~nm}$.

\section{Discussion}

The control of $g_{Q E P}$ of the coupling systems also has significant influences on the quantum coherence properties in the plasmon-exciton coupling systems. As is well known that when the interaction is in the strong coupling regime, plasmon and exciton will give up their separate identities and the hybrid quasiparticles of plexciton can be formed, which is also a reflection of the quantum coherence between these two components. Figure 5a shows the plasmonic and exciton fractions in the upper (UPB) and lower (LPB) plexciton branch of the single-exciton-coupled NRs located on the FP cavity and the ITO substrate, respectively. Compared to that of the singleexciton-coupled NRs on ITO substrate, the plasmonic and exciton fractions in plexciton branches show significant coherence (or mixed) characteristics in the coupled NRs engineered by the leaky FP cavity, which is induced by successfully lowering the $g_{Q E P}$ of the hybrid systems. On the other hand, an improved cooperativity of coherence $C \sim 1.92$, corresponding to the $\beta$ factor of $\sim 79.3 \%$, can also be obtained from the strong coupling case in Fig. 4a, which is more than two times larger than that $(\sim 0.83)$ of the weak coupling case shown in Fig. 1b. To the best of our knowledge, this cooperativity is the maximum for single-exciton strong coupling with plasmon modes at room temperature ${ }^{4,26-29}$. In Fig. 5b, we list the statistics of $C$ for the single-exciton strong coupling with different NRs. An average cooperativity $\bar{C} \sim 2.04$ is observed, which is much larger than those $(\sim 0.85)$ obtained from the single-exciton-coupled NRs without FP-cavity engineering. Noteworthy, many important applications such as generation of single photons on demand ${ }^{51}$, the construction of single-photon switches ${ }^{52}$, etc. require $C>1^{53}$, a regime of cavity quantum electrodynamics in which an optical emitter radiates with high probability into a distinct light mode ${ }^{54}$. The improved 
cooperativities also provide possibilities for the FP-cavity engineered single-exciton-coupled NRs in exploiting the high-fidelity quantum gates operated at ambient conditions ${ }^{55}$.
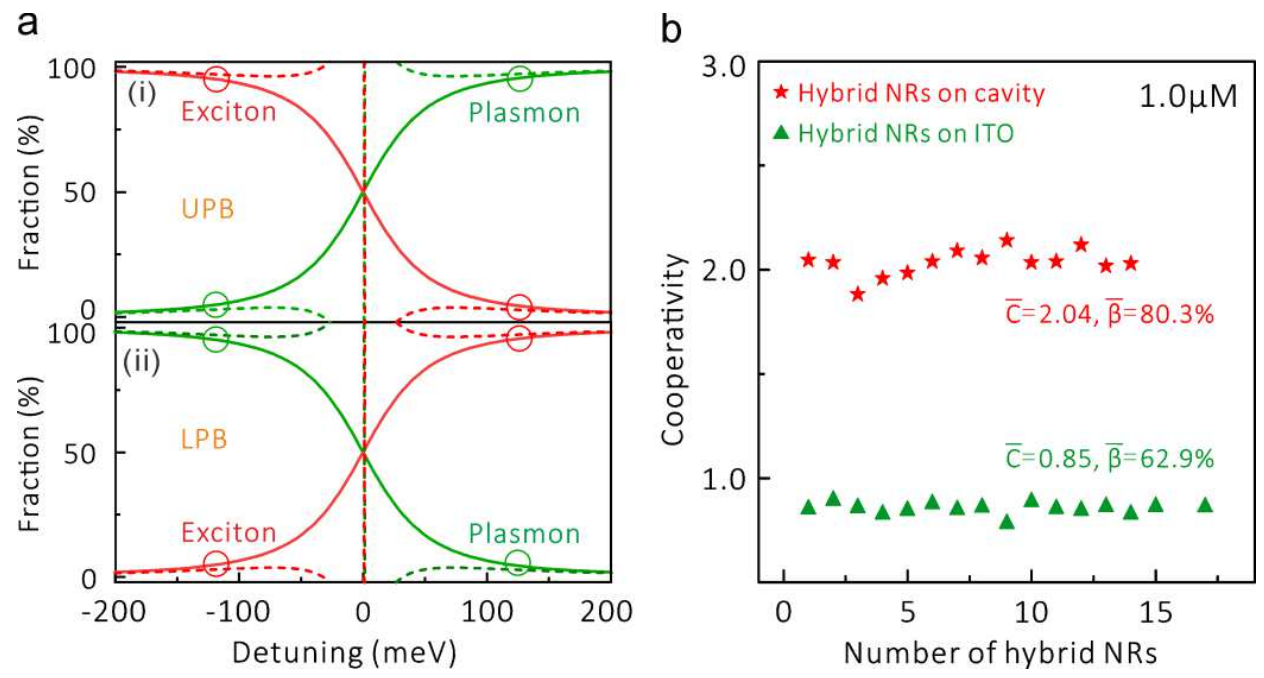

Figure 5 | Influence of controlling $g_{Q E P}$ on coherence properties in plasmon-exciton coupling systems. a Plasmonic and exciton fractions for the UPB and LPB of the single-exciton-coupled NRs located on the FP cavity (solid color curves) and the ITO substrate (dashed color curves), In the calculations, the parameters are the same as what have been used in Figs. 1 d and $4 \mathbf{e}$. b Cooperativities of the individual single-exciton-coupled NRs located on the FP cavity (red stars) and the ITO substrate (green triangles), which are calculated from the experimental data in Fig. 1c and Fig. 4e, respectively.

In summary, we have revealed that the high critical coupling energy at the QEP due to large mismatch between the dissipative decays of the QE and LPM seriously hinders the realization of the single-QE strong coupling at room temperature. It is theoretically and experimentally demonstrated that the good decay match between the two coupling subsystems can be achieved by suppressing the dissipative decay of the LPM with use of a leaky Fabry-Perot cavity, resulting in a considerable decrease of the critical coupling-energy at the QEP. The proposed strategy gets rid of the extreme conditions (such as cryogenic temperatures and ultrahigh vacuum, or ultrasmall 
mode volume) previously required by the single-QE strong coupling with photonic modes, which makes its realization at room temperature much easier. Our work not only opens a new path to easily realize the room-temperature single-QE strong coupling with photonic modes, but also provides a flexible way to control and access the QEP in ambient conditions.

\section{Methods}

Theoretical analysis. Please see the Supplementary Note 2 for the derivations and discussions.

Simulations. The simulation results were performed using a commercial software, COMSOL Multiphysics. To better calculate the scattering problem of nanoparticle (NP) placed on a substrate, two Electromagnetic Waves, Frequency Domain interfaces were used. In the first interface, periodic (Floquet) boundary conditions were applied in the $x$ and $y$ directions, while two Port conditions (one defines the incident plane wave and allows for specular reflection, the other absorbs the transmitted plane wave.) were applied in the $z$ direction. A second interface introduces the NP as the scatterer and surrounds the geometry with PMLs.

Materials. Cetyltrimethylammonium bromide $(\mathrm{CTAB} \geq 99 \%)$, benzyldimethyldodecylammonium chloride (BBDAC, 99\%), cetyltrimethylammonium chloride (CTAC, 99\%), gold chloride trihydrate $\left(\mathrm{HAuCl}_{4} \cdot 3 \mathrm{H}_{2} \mathrm{O},>99 \%\right)$, silver nitrate $\left(\mathrm{AgNO}_{3}, 99.8 \%\right)$, sodium borohydride $\left(\mathrm{NaBH}_{4}, 99 \%\right), 1$-ascorbic acid (AA, $\left.\mathrm{C}_{6} \mathrm{H}_{8} \mathrm{O}_{6},>99 \%\right)$, sodium hydroxide $(\mathrm{NaOH}, \geq 98 \%), 5,6$-Dichloro-2- [[5,6-dichloro-1-ethyl-3-(4sulfobutyl)-benzimidazol-2-ylidene]-propenyl]-1-ethyl-3-(4-sulfobutyl)-benzimidazolium hydroxide, inner salt, sodium salt (TDBC dye) were purchased from Sigma Aldrich and used as received. In all experiments, we used deionized water (DI) with a resistivity of $18.25 \mathrm{M} \Omega . \mathrm{cm}$.

Sample fabrication. Au NRs were synthesized by a seed mediated growth process as reported previously ref.

${ }^{28}$. Briefly, the seed solution was prepared by the rapid addition of an ice-cold $\mathrm{NaBH}_{4}$ solution $(0.6 \mathrm{~mL}, 0.01 \mathrm{M})$ into a mixture solution composed of $\mathrm{HAuCl}_{4}(25 \mathrm{uL}, 0.1 \mathrm{M})$ and CTAB $(10 \mathrm{~mL}, 0.1 \mathrm{M})$. The resultant solution was vigorously stirred for $5 \mathrm{~min}$ then kept at $28{ }^{\circ} \mathrm{C}$ for $2 \mathrm{~h}$ to nucleate seed. The growth solution was prepared by the sequential addition of CTAB $(25 \mathrm{~mL}, 0.2 \mathrm{M})$, BBDAC (2.5 mL, $0.2 \mathrm{M}), \mathrm{HAuCl}_{4}(2.5 \mathrm{~mL}, 0.01 \mathrm{M})$, 
$\mathrm{AgNO}_{3}(0.04 \mathrm{~mL}, 0.1 \mathrm{M}), \mathrm{AA}(0.325 \mathrm{~mL}, 0.1 \mathrm{M})$ into $25 \mathrm{~mL}$ DI. $60 \mu \mathrm{L}$ seed solution was added into the growth solution, stirred for $2 \mathrm{~min}$ and left undisturbed in a $28^{\circ} \mathrm{C}$ water-bath for over 15 hours. The TEM image and extinction spectrum of the purified Au NRs were shown in Supplementary Fig. 14.

$\mathrm{Au} @ \mathrm{Ag}$ NRs were prepared by coating Ag nanoshell on the Au NRs, which would lead to appearance of a hypsochromically-shifted extinction band. The thicker the shell grows, the further the extinction spectrum shifts. To fabricate the cuboid $\mathrm{Au} @ \mathrm{Ag}$ NRs, 10mL of the as-made Au NRs were centrifuged under 7500 rpm for 20 minutes. After removing the supernatant, we carefully redispersed the dark green sediment into a CTAC solution $(10 \mathrm{~mL}, 0.08 \mathrm{M})$. Five aliquots $(2 \mathrm{~mL})$ of the as-prepared Au NRs were transferred into five clean bottles. Different amount of $0.01 \mathrm{M} \mathrm{AgNO}_{3}$ were added to each aliquot, following by the $0.1 \mathrm{M}$ AA solutions. The volume of the $\mathrm{AgNO}_{3}$ solutions were twice as much as the AA solutions. The mixed solutions were left in a 65 ${ }^{\circ} \mathrm{C}$ water-bath for 4 hours. Finally, all aliquots were centrifuged twice $(7500 \mathrm{rpm}, 20 \mathrm{~min}$ ) and redispersed in 5 mL DI respectively. The typical TEM image and extinction spectrum of the prepared Au@Ag NRs with resonance wavelength of $\sim 590 \mathrm{~nm}$ were shown in Supplementary Fig. 15.

The Au@Ag NR/J-aggregate hybrids were fabricated by electrostatically assembling J-aggregates of TDBC dye molecules on individual Au@Ag NR. Before the fabrication of Au@Ag NR/J-aggregate hybrids, TDBC Jaggregates were firstly prepared by adding $5 \mathrm{mg} \mathrm{NaOH}$ to $1 \mathrm{~mL} 10^{-4} \mathrm{M}$ TDBC dye monomer solution. After that, two Au@Ag NR/J-aggregate hybrid samples were fabricated by injecting 10 and $30 \mu \mathrm{L}$ TDBC J-aggregates into the as-prepared colloidal $\mathrm{Au} @ \mathrm{Ag}$ NR sample of $1 \mathrm{~mL}$, respectively. After vigorous stirring the mixture for 10 min, the resulting colloidal solution was centrifugated $(4000 \mathrm{rpm}, 10 \mathrm{~min})$, and the unbound J-aggregates and monomers were removed. Finally, we resuspended the depositions (the Au@Ag NR/J-aggregate hybrids) in 2 $\mathrm{mL}$ DI for next measurements. Note that, one can control the exciton number involved in the plasmon-exciton coupling via controlling the amount of the added J-aggregates in the colloidal Au@Ag NRs (1 mL).

The leaky Fabry-Perot (FP) cavities constructed by three dielectric layers of $\mathrm{Si} / \mathrm{SiO}_{2} / \mathrm{Si}_{3} \mathrm{~N}_{4}$ were fabricated using the method of inductively coupled plasma chemical vapor deposition (ICPCVD, PlasmaPro System100 ICP180-CVD, Oxford, UK). The schematic of the fabrication process can be found in Supplementary Fig. 16. Specifically, 3000-nm thick stoichiometric $\mathrm{SiO}_{2}$ on the single crystal silicon substrate was firstly deposited, next, a 200-nanometer film of silicon nitride $\left(\mathrm{Si}_{3} \mathrm{~N}_{4}\right)$ was deposited in the same way. After these two simple processes, 
the FP cavity sample was constructed. After the measurement using the thin-film analyzer (Filmetrics, F20$\mathrm{UV} / \mathrm{F} 20-\mathrm{UVX} / \mathrm{F} 20 / \mathrm{F} 20-\mathrm{EX}, \mathrm{USA})$, we found that the final thickness of $\mathrm{SiO}_{2}\left(\mathrm{Si}_{3} \mathrm{~N}_{4}\right)$ was $\sim 3008 \mathrm{~nm}(\sim 217 \mathrm{~nm})$, and the surface of the silicon nitride structure was found to be rather flat and smooth. It is worth pointing out that the sandwich $\mathrm{Si}_{3} \mathrm{~N}_{4} / \mathrm{SiO}_{2} / \mathrm{Si}$ slab cavity structure developed here is particularly robust. Unlike typical plasmonic cavities which surface or shell is oxidized with time, almost no performance degradation is observed even up to one year. After the FP cavity fabricated, individual Au@Ag NR/J-aggregate hybrids were dropped on the up surface of the cavity for next dark-filed scattering measurements.

Optical and morphology characterization. Extinction spectra were measured on an ultraviolet-visible-nearinfrared spectrometer (Lambda 950, PerkinElmer) with a high spectral resolution of $0.08 \mathrm{~nm}$. The TEM images were taken by a 2010HT TEM machine (JEOL Inc., Japan) operated at $100 \mathrm{kV}$. The high-resolution TEM images were obtained by Titan G2 60-300 (FEI Inc., America) microscope operated at 300 kV. The SEM images were taken by Auriga-39-34 (Zeiss Inc., Germany) microscope operating at $5.0 \mathrm{kV}$.

Dark-filed scattering measurements. The schematic of our dark-filed scattering measurements can be referred in ref. ${ }^{28}$. Before the scattering measurements, $25 \mu \mathrm{L}$ of the Au@Ag NR/J-aggregates bulk solution was dropcasted onto the up surface of the FP cavity (or the ITO coated glass substrate). After about one min, the droplet was removed, and the samples were washed with deionized water and dried under nitrogen conditions. Then, the individual Au@Ag NR/J-aggregate hybrids isolated from the sample could be formed and fixed on the surface of FP cavity (or the ITO-coated glass substrate). Note that, the ITO material has a reflex index very close to the $\mathrm{Si}_{3} \mathrm{~N}_{4}$ in the visible spectral range, but has a much better electrical conductivity which allows the Au@Ag NRs to be well characterized with both SEM and dark-field imaging). In order to obtain the scattering spectroscopy of the individual hybrids, we combined dark-filed microscopy with SEM of the same field of view to correlate elastic scattering spectra and structures on the level of single hybrid NR. The scattering images and spectra of the individual Au@Ag NR/J-aggregate hybrids were recorded on a dark-field optical microscope (Olympus BX51, Olympus Inc.) that was integrated with a monochromator (Acton Spectra Pro 2360, Acton Inc.), a quartz tungsten halogen lamp $(100 \mathrm{~W})$, and a charge-coupled device camera (Princeton Instruments Pixis 400BR_eXcelon). To obtain clean scattering signals from a single isolated hybrid NR, the commercial 
spectrograph equipped with an entrance slit was set at $\sim 100 \mathrm{~nm}$ and the camera was cooled to $-70^{\circ} \mathrm{C}$ during the measurements.

For collection of the scattering spectra of the individual hybrids, the light was launched from a dark-field objective (100×, numerical aperture 0.80$)$, and the light scattered in the backward direction was collected by the same objective. The color scattering images were captured using a color digital camera (ARTCAM-300MI-C, ACH Technology Inc.) mounted on the imaging plane of the microscope.

\section{Data availability}

The data that support the plots within this paper and other findings of this study are available from the corresponding author upon reasonable request.

\section{References}

1. Tiecke, T. G. et al. Nanophotonic quantum phase switch with a single atom. Nature 508, 241-244 (2014).

2. McKeever, A. B. J., Boozer, A. D., Buck, J. R. \& Kimble, H. J. Experimental realization of a one-atom laser in the regime of strong coupling. Nature 425, 268-271 (2003).

3. Imamoglu, A. et al. Quantum Information Processing Using Quantum Dot Spins and Cavity QED. Phys. Rev. Lett. 83, 4204-4027 (1999).

4. Chang, D. E., Vuletić, V. \& Lukin, M. D. Quantum nonlinear optics-photon by photon. Nature Photon. 8, 685-694 (2014).

5. Khitrova, G., Gibbs, H. M., Kira, M., Koch, S. W. \& Scherer, A. Vacuum Rabi splitting in semiconductors. Nature Phys. 2, 81-90 (2006).

6. Seskir, Z. C. \& Aydinoglu, A. U. The Landscape of Academic Literature in Quantum Information Technologies. Preprint at $<$ https://arxiv.org/abs/1910.06969> (2019).

7. Hennessy, K. et al. Quantum nature of a strongly coupled single quantum dot-cavity system. Nature 445, 896899(2007).

8. Törmä, P. \& Barnes, W. L. Strong coupling between surface plasmon polaritons and emitters: a review. Rep Prog Phys.78, 013901 (2015). 
9. Groß, H., Hamm, J. M., Tufarelli, T., Hess, O. \& Hecht, B. Near-field strong coupling of single quantum dots. Sci. Adv 4: eaar4906 (2018).

10. Vahala, K. J. Optical microcavities. Nature 424, 839-846 (2003).

11. Benson, O. Assembly of hybrid photonic architectures from nanophotonic constituents. Nature 480, 193-199 (2011).

12. Kimble, H. J. The quantum internet. Nature 453, 1023-1030 (2008).

13. Aoki, T. et al. Observation of strong coupling between one atom and a monolithic microresonator. Nature 443, 671-674 (2006).

14. Thompson, R. J., Rempe, G. \& Kimble, H. J. Observation of normal-mode splitting for an atom in an optical cavity. Phys. Rev. Lett. 68, 1132-1135(1992).

15. Groblacher, S. et al. An experimental test of non-local realism. Nature 446, 871-875 (2007).

16. Reithmaier, J. P. et al. Strong coupling in a single quantum dot-semiconductor microcavity system. Nature 432, 197-200 (2004).

17. Englund, D. et al. Controlling cavity reflectivity with a single quantum dot. Nature 450, 857-861 (2007).

18. Bellessa, J., Bonnand, C., Plenet, J. C. \& Mugnier, J. Strong coupling between surface plasmons and excitons in an organic semiconductor. Phys. Rev. Lett. 93, 036404 (2004).

19. Hakala, T. K. et al. Vacuum Rabi splitting and strong-coupling dynamics for surface-plasmon polaritons and rhodamine 6G molecules. Phys. Rev. Lett. 103, 053602 (2009).

20. Waks, E. \& Sridharan, D. Cavity QED treatment of interactions between a metal nanoparticle and a dipole emitter. Phys. Rev. A 82, 043845 (2010).

21. Gomez, D. E., Vernon, K. C., Mulvaney, P. \& Davis, T. J. Surface plasmon mediated strong exciton-photon coupling in semiconductor nanocrystals. Nano Lett. 10, 274-278 (2010).

22. Savasta, S. et al. Nanopolaritons: Vacuum Rabi Splitting with a Single Quantum Dot in the Center of a Dimer Nanoantenna. ACS Nano 4, 6369-6376 (2010).

23. Schlather, A. E., Large, N., Urban, A. S., Nordlander, P. \& Halas, N. J. Near-field mediated plexcitonic coupling and giant Rabi splitting in individual metallic dimmers. Nano Lett. 13, 3281-3286 (2013).

24. Zengin, G. et al. Realizing Strong Light-Matter Interactions between Single-Nanoparticle Plasmons and 
Molecular Excitons at Ambient Conditions. Phys. Rev. Lett. 114, 157401 (2015).

25. Ren, J. et al. Evanescent-Vacuum-Enhanced Photon-Exciton Coupling and Fluorescence Collection. Phys. Rev. Lett. 118, 073604 (2017).

26. Santhosh, K., Bitton, O., Chuntonov, L. \& Haran, G. Vacuum Rabi splitting in a plasmonic cavity at the single quantum emitter limit. Nature Commun.7, 11823 (2016).

27. Chikkaraddy, R. et al. Single-molecule strong coupling at room temperature in plasmonic nanocavities. Nature 535, 127-130 (2016).

28. Liu, R. et al. Strong Light-Matter Interactions in Single Open Plasmonic Nanocavities at the Quantum Optics Limit. Phys. Rev. Lett. 118, 237401 (2017).

29. Leng, H., Szychowski, B., Daniel, M. C. \& Pelton, M. Strong coupling and induced transparency at room temperature with single quantum dots and gap plasmons. Nature Commun. 9, 4012 (2018).

30. Naghiloo. M. et al. Quantum state tomography across the exceptional point in a single dissipative qubit. Nature Phys. 15, 1232-1236 (2019).

31. Wu, Y. et al. Observation of parity-time symmetry breaking in a single-spin system. Science 364, 878-880 (2019).

32. Klauck, F. et al. Observation of PT-symmetric quantum interference. Nature Photon. 13, 883-887 (2019).

33. Chen, H. -Z. et al. Revealing the missing dimension at an exceptional point. Nature Phys. 16, 571-578 (2020).

34. Jiang, Y. et al. Resonance Coupling in an Individual Gold Nanorod-Monolayer $\mathrm{WS}_{2}$ Heterostructure: Photoluminescence Enhancement with Spectral Broadening. ACS Nano, 0c06220 (2020).

35. Saitoh, E. et al. Current-induced resonance and mass determination of a single magnetic domain wall. Nature 432, 200-203 (2004).

36. Manjavacas, A., García de Abajo, F. J. \& Nordlander, P. Quantum plexcitonics: strongly interacting plasmons and excitons. Nano Lett. 11, 2318-2323 (2011).

37. Liu, R, Yu, Y. -C. \& Wang, X.-H. Relativity and diversity of strong coupling to measured subsystems. Preprint at < https://arxiv. org/abs/2003. $06982>(2020)$. 
38. Limonov, M. F., Rybin, M. V., Poddubny, A. N. \& Kivshar, Y. S. Fano resonances in photonics. Nature Photon. 11, 543-554 (2017).

39. Doeleman, H. M., Verhagen, E. \& Koenderink, A. F. Antenna-Cavity Hybrids: Matching Polar Opposites for Purcell Enhancements at Any Linewidth. ACS Photonics 3, 1943-1951 (2016).

40. Yin, Y. et al. Localized Surface Plasmons Selectively Coupled to Resonant Light in Tubular Microcavities. Phys. Rev. Lett. 116, 253904 (2016).

41. Peng, P. et al. Enhancing Coherent Light-Matter Interactions through Microcavity-Engineered Plasmonic Resonances. Phys. Rev. Lett. 119, 233901 (2017).

42. Gazzano, O. et al. Evidence for confined tamm plasmon modes under metallic microdisks and application to the control of spontaneous optical emission, Phys. Rev. Lett. 107, 247402 (2011).

43. Barth, M. et al. Nanoassembled plasmonic-photonic hybrid cavity for tailored light-matter coupling, Nano Lett. 10, 891-895 (2010).

44. Ai, Q. et al. Ultranarrow Second-Harmonic Resonances in Hybrid Plasmon-Fiber Cavities, Nano Lett. 18, $5576-5582(2018)$.

45. Angelis, F. D. et al. A Hybrid Plasmonic-Photonic Nanodevice for Label-Free Detection of a Few Molecules. Nano Lett. 8, 2321 (2008).

46. Gu, F., Zhang L., Zhu Y., \& Zeng, H. Free-space coupling of nanoantennas and whispering-gallery microcavities with narrowed linewidth and enhanced sensitivity. Laser Photonics Rev. 9, 682-688 (2015).

47. Dantham, V. R. et al. Label-free detection of single protein using a nanoplasmonic-photonic hybrid microcavity, Nano Lett., 13, 3347-3351 (2013).

48. González-Rubio, G. et al. Femtosecond laser reshaping yields gold nanorods with ultranarrow surface plasmon resonances. Science 358, 640-644 (2017).

49. Wang, Y. et al. Stable, high-performance sodium-based plasmonic devices in the near infrared. Nature 581, 401-405 (2020).

50. Vasista, A. B. \& Barnes, W. L. Molecular Monolayer Strong Coupling in Dielectric Soft Microcavities. Nano Lett. 20, 1766-1773 (2020).

51. He, Y-M. et al. On-demand semiconductor single-photon source with near-unity indistinguishability. Nature 
Nanotech. 8, 213-217 (2013).

52. Volz, T. et al. Ultrafast all-optical switching by single photons. Nature Photon. 6, 605-609 (2012).

53. Stehle, C., Zimmermann, C. \& Slama, S. Cooperative coupling of ultracold atoms and surface plasmons. Nature Phys. 10, 937-942 (2014).

54. Kimble, H. J. Strong interactions of single atoms and photons in cavity qed. Phys. Scr. T 76, 127-137 (1998).

55. Najer, D. et al. A gated quantum dot strongly coupled to an optical microcavity. Nature 575, 622-627（2019）

\section{Acknowledgments}

This work was supported by the National Key R\&D Programs of China (Grant No. 2016YFA0301300), the National Natural Science Foundations of China (Grant Nos. 11874438, 91750207 and 11761141015), the KeyArea Research and Development Program of Guangdong Province (Grant No.2018B030329001), the Natural Science Foundations of Guangdong (Grant Nos. 2016A030312012 and 2018A030313722), the Guang Zhou Science and Technology Projects (201607020023) and the Open Fund of IPOC (BUPT) (Grant No. IPOC2018B007).

\section{Author contributions}

X.H.W. presented the idea and supervised the project. X.H.W., R.M.L. W.L. conceived the strategy for the experimental design and data analysis. W.L., R.M.L. and J.Y.L. performed the sample preparations. W.L. performed the numerical simulations. W.L., R.M.L. and H.J.C. performed the optical measurements. J.Z. and H.J.C. contributed to the preparation of the experimental setup and measurement. R.M.L. and X.H.W. cowrote the paper. All authors discussed the results and commented on the manuscript.

\section{Competing interests}

The authors declare that they have no competing financial interests.

\section{Additional information}

Supplementary information is available in the online version of the paper. Reprints and permissions information is available online at www.nature.com/reprints. Correspondence and requests for materials should 
be addressed to X.H.W. 
a

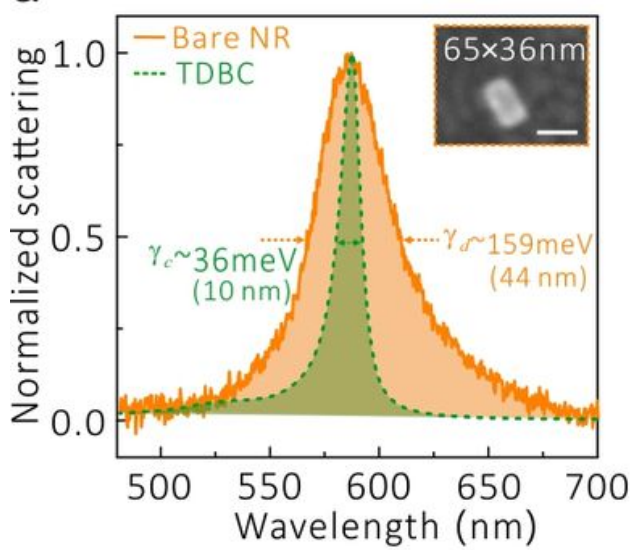

b

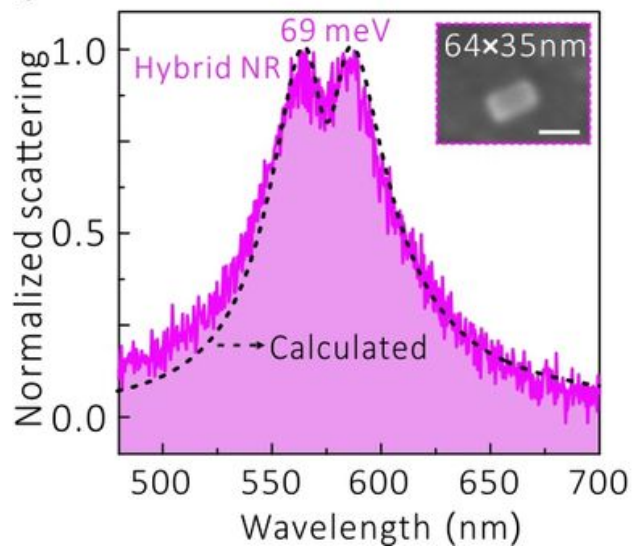

C

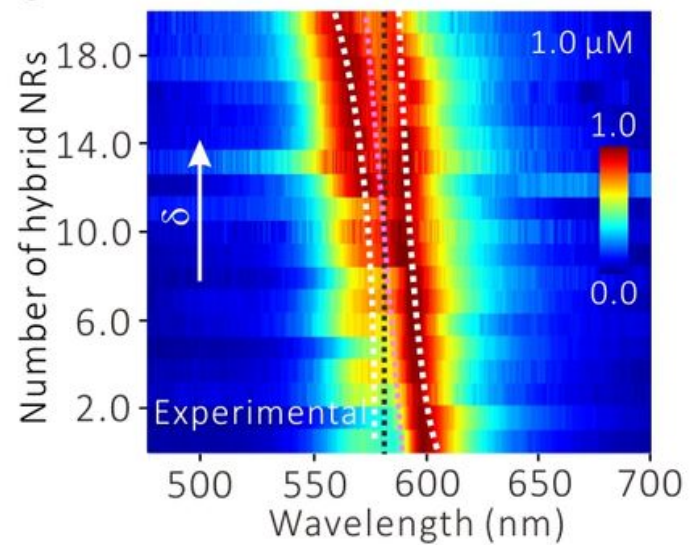

d

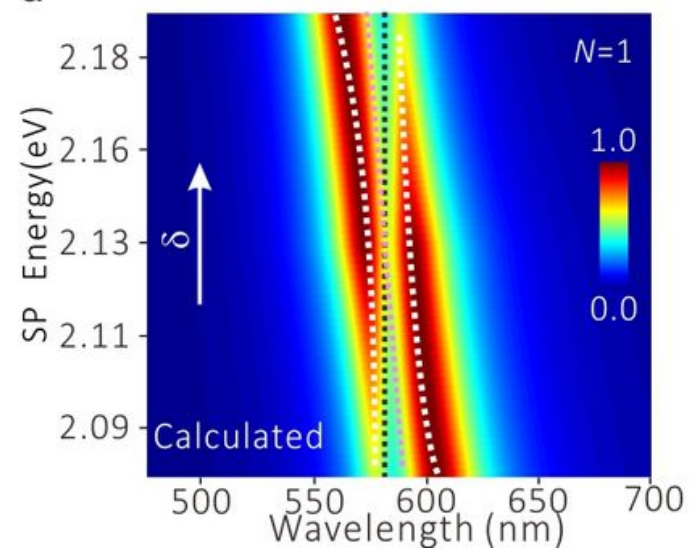

e

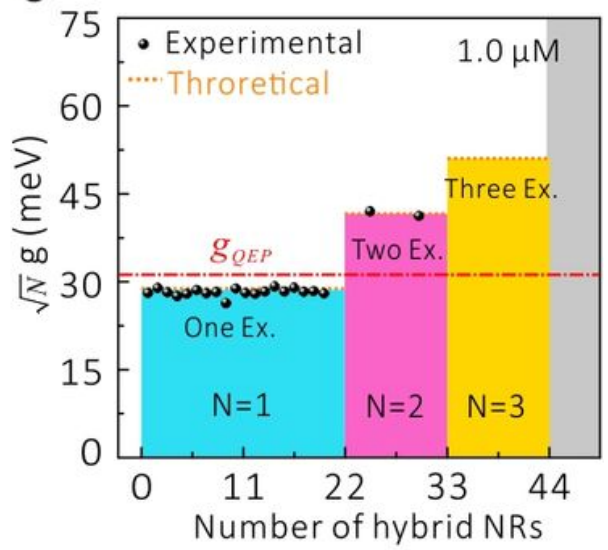

f

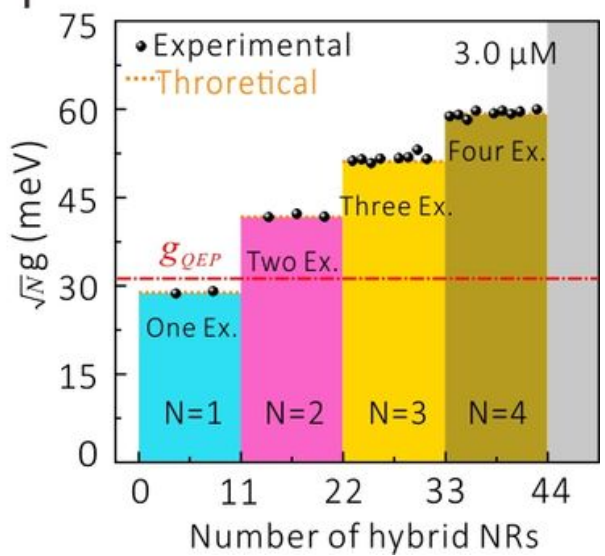

Figure 1

LPM-exciton couplings in single Au@Ag NR/J-aggregate hybrids on ITO substrate. a Normalized scattering of a bare Au@Ag NR resonant to the exciton absorption (dashed green curve) with $\varepsilon c 2.11$ meV. b Normalized scattering spectra of a strongly coupled Au@Ag NR/J-aggregate hybrid at resonance condition. The dotted black line is the theoretical result calculated using Eq. (3) with $N=1$. c Normalized scattering spectra of the individual Au@Ag NR/J-aggregate hybrids isolated from the ensembles treated with 1.0- $\mu$ Mdye solution, and ordered according to the detuning. $d$ Extinction spectra calculated using Eq. (3) with $\mathrm{N}=1$ for the same samples as in (c). e, f Quantum step statistics of the effective coupling strength $(\sqrt{ } \mathrm{Ng})$ extracted (using the method proposed in ref. 37.) from the measured individual hybrids isolated from the ensembles treated with (e) $1.0-\mu \mathrm{M}$ and (f) $3.0-\mu \mathrm{M}$ dye solution, respectively. The insets in (a), (b) are SEM images of the measured nanostructures, and the scale bar is $50 \mathrm{~nm}$. 
a

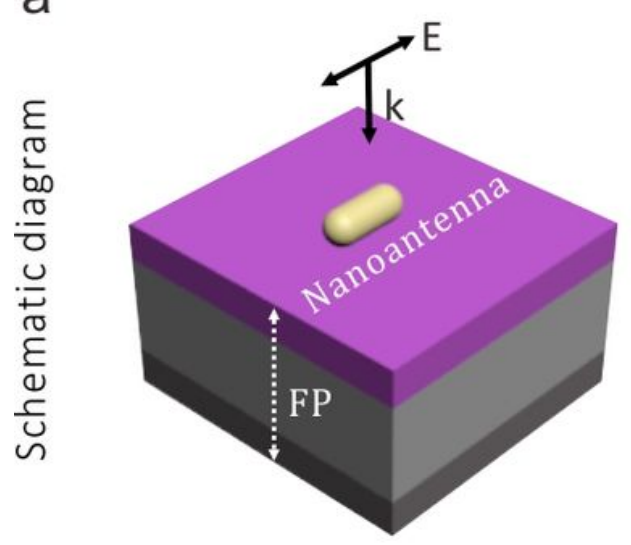

Si $\mathrm{SiO}_{2}$ $\mathrm{Si}_{3} \mathrm{~N}_{4}$ $\mathrm{Au}$

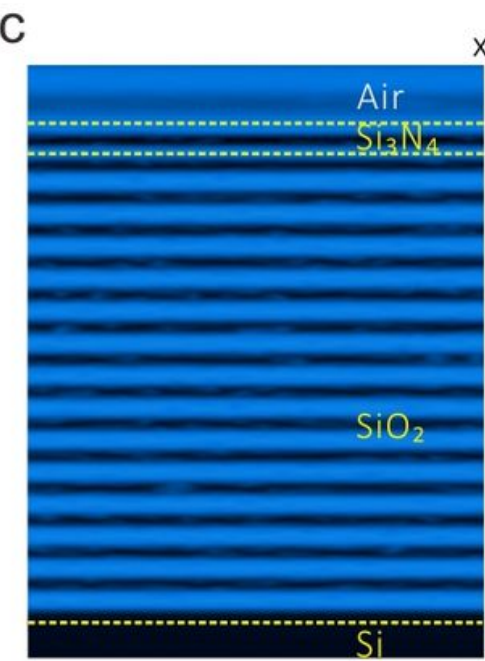

b

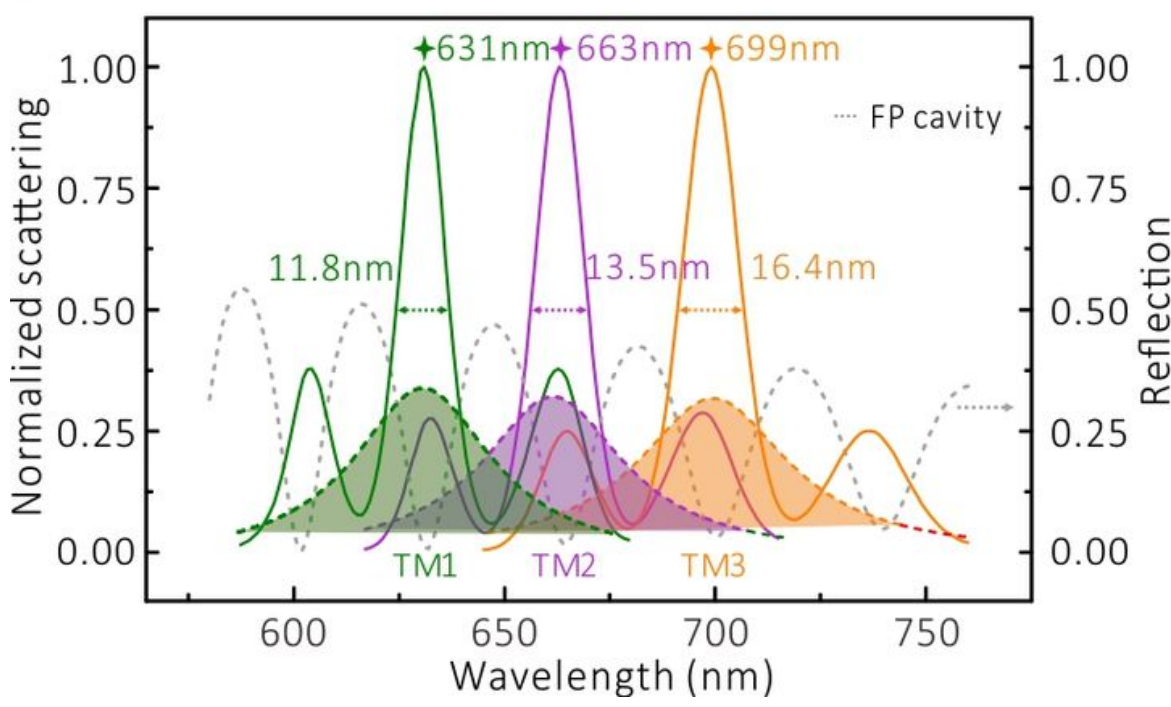

$\times 10^{5} \mathrm{~V} / \mathrm{m} \mathrm{d}$ 1.4

. 0

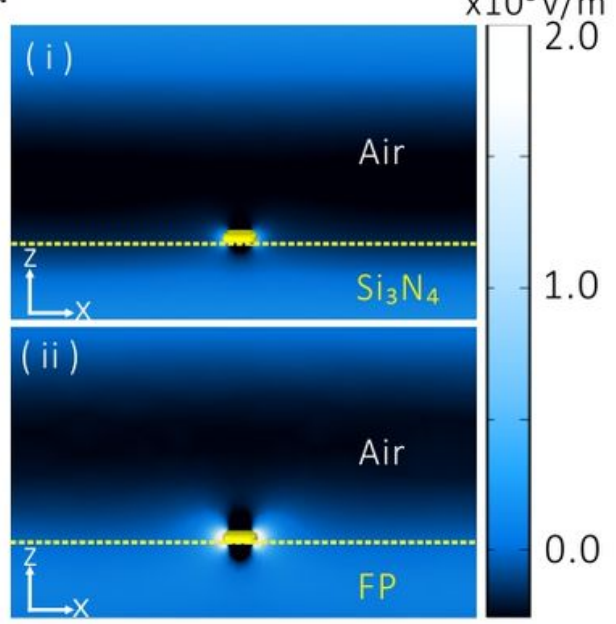

0.6

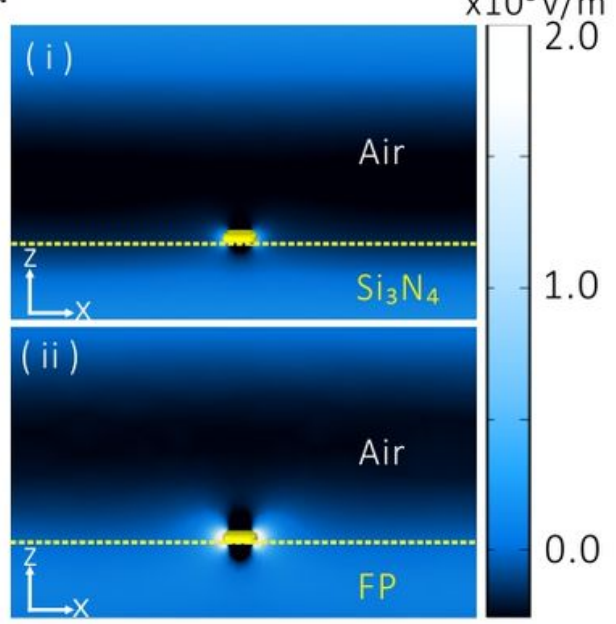

0.2

e

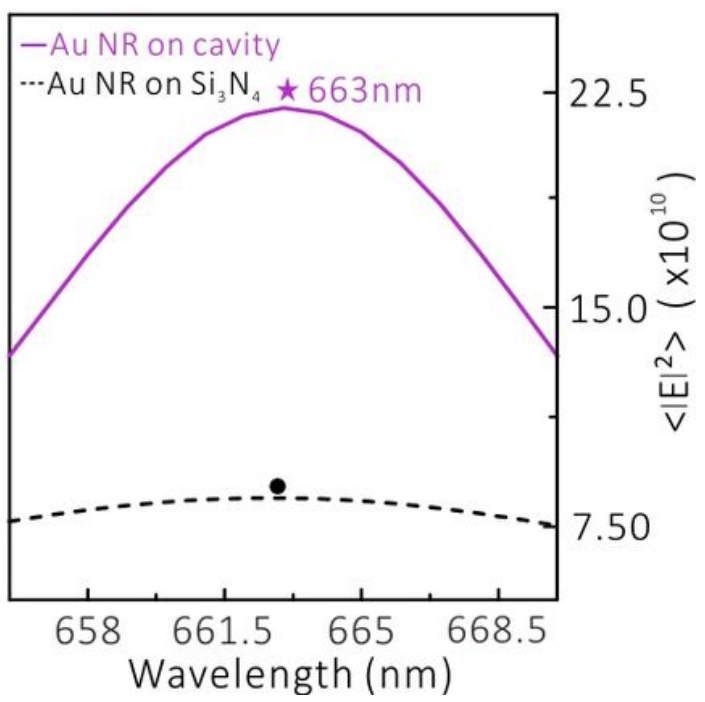

Figure 2

Synergistic nanoantenna-microcavity hybrid for plasmonic decay suppression and local EF enhancement. a Schematic diagram of the synergistic Au NR/FP-cavity hybrid system. b Normalized scattering calculated for the Au NRs with different aspect ratios localized on the surface of the leaky FP cavity. The solid and dashed color curve represents the normalized scattering of the Au NRs on the FP cavity and on the bulk Si3N4 substrate, respectively. The dashed gray curve is the reflectivity of the bare FP cavity. Here, the length I and radius $r$ of the Au NR are 45.2, 54.0, 61.8 and $12 \mathrm{~nm}$, respectively; c Simulated total EF through the middle of the bare FP cavity. $d$ Simulated $x$-component of the total EF, Ex, through the middle of the Au NR (I =54 nm) on the bulk Si3N4 substrate (i) and the FP cavity (ii), respectively. e Average near-field intensity $邓 I E I 2 \rrbracket o n$ the surface of the Au NR $(I=54 \mathrm{~nm})$ as functions of wavelength for the NR located on the bulk Si3N4 substrate and the FP cavity, respectively. In simulations, tSi3N4 = $200 \mathrm{~nm}$, tSiO2 = $4035 \mathrm{~nm}$, silica is the substrate. 
a
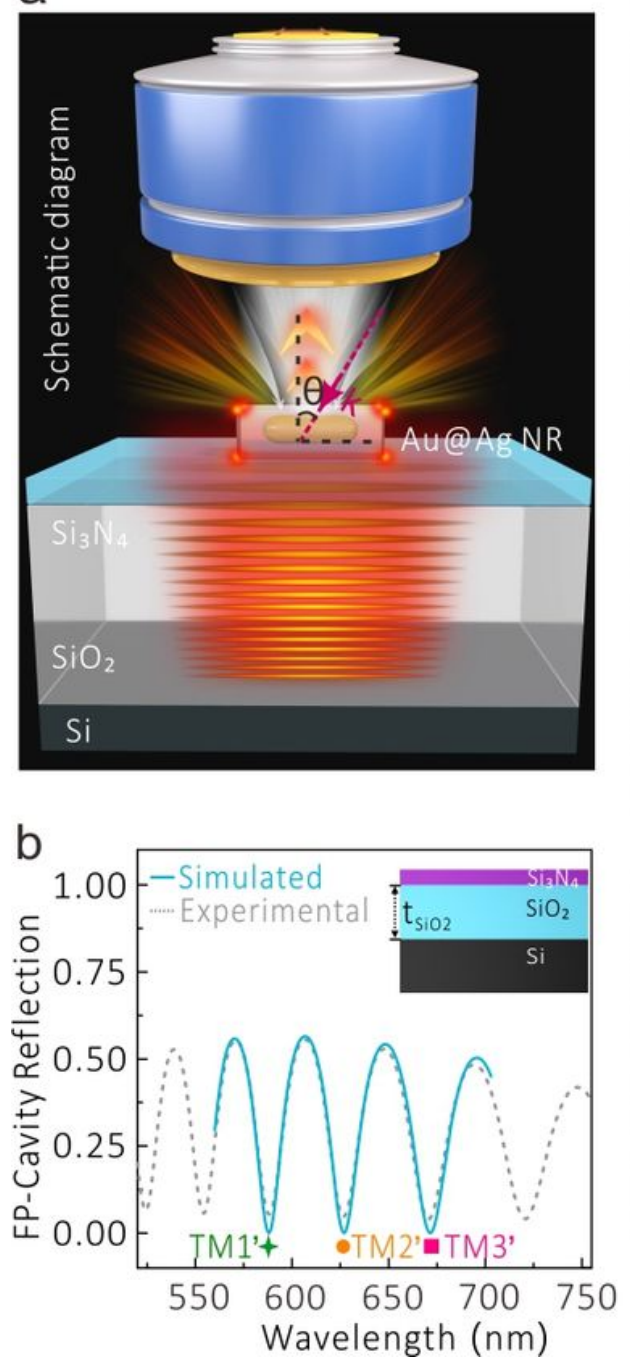

C
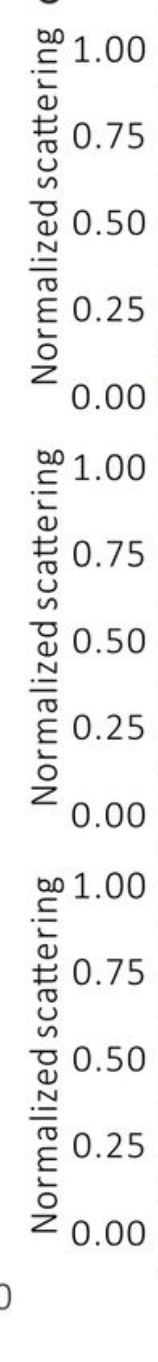

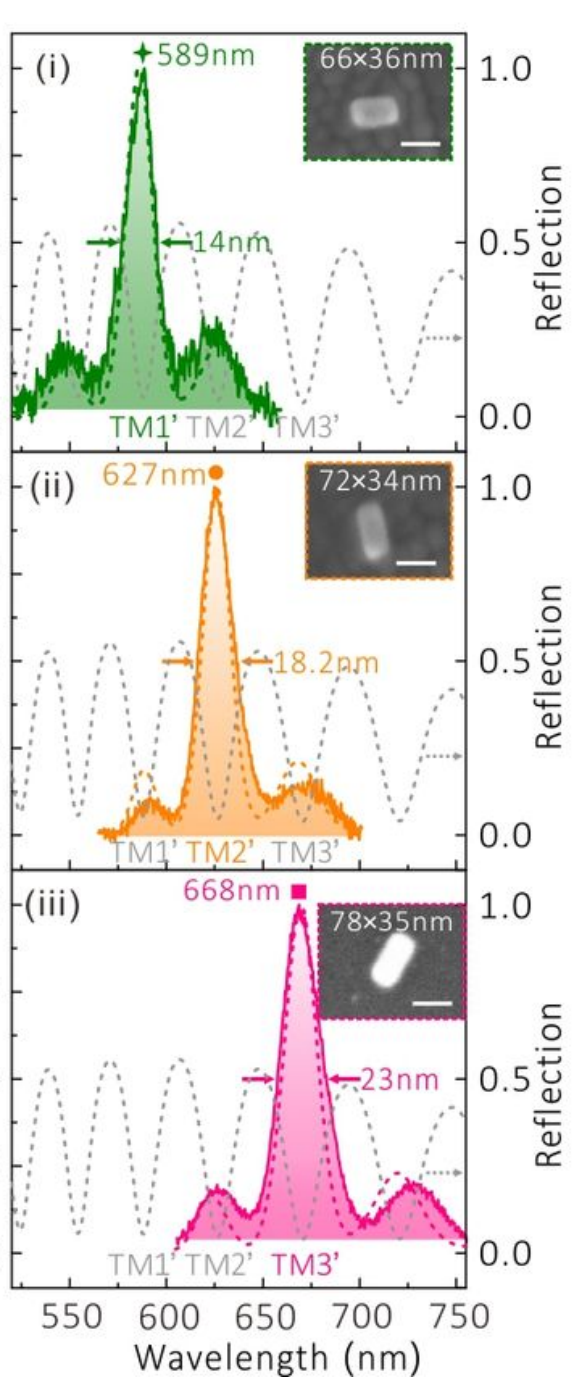

d
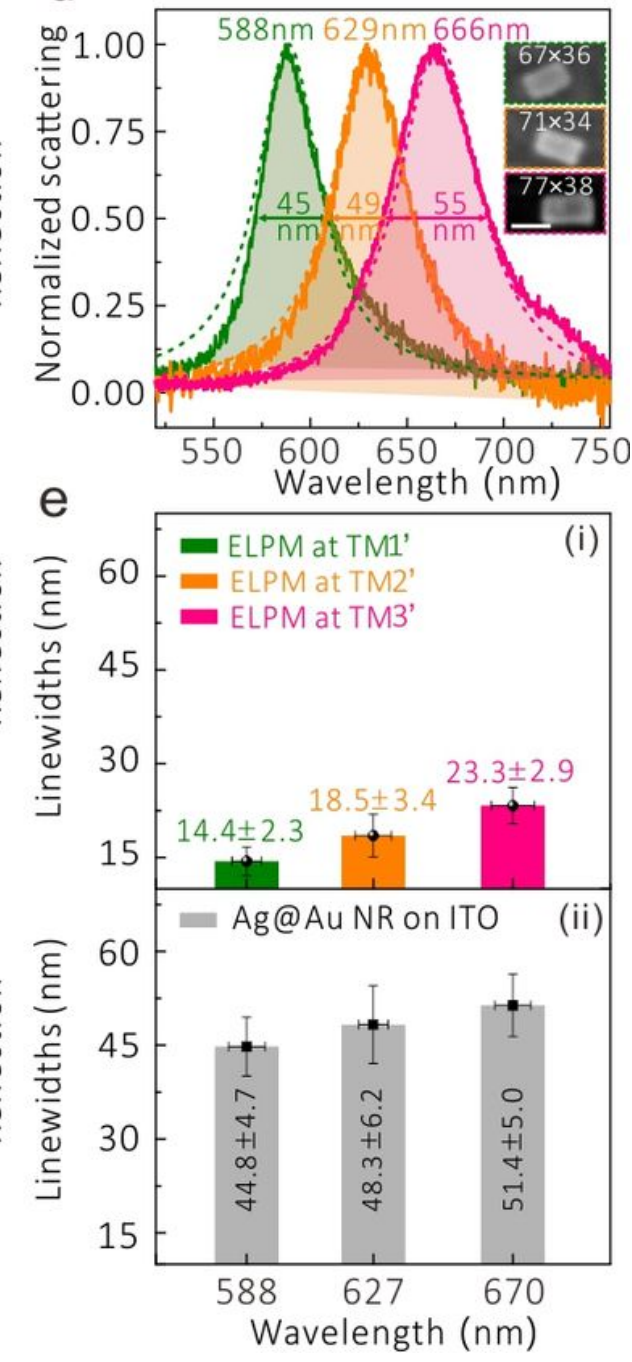

Figure 3

Scattering measurements of the bare Au@Ag NRs in the EM environments engineered by the leaky FP cavity. a Schematic diagram of dark-field scattering measurement for a single cuboid Au@Ag NR on a leaky FP cavity. b Simulated (solid cyan curve) and measured (dashed grey line) reflectance for the leaky FP cavity with $\mathrm{tSi} 3 \mathrm{~N} 4=216.9 \mathrm{~nm}$ and $\mathrm{tSiO} 2=3008 \mathrm{~nm}$. The silicon is set as a semi-infinite substrate. $\mathrm{c}, \mathrm{d}$ Normalized scattering (solid color curves) for Au@Ag NRs located on (c) the FP cavity (with tSi3N4 = $216.9 \mathrm{~nm}$ and $\mathrm{tSiO} 2=3008 \mathrm{~nm}$ ) and (d) the ITO-coated glass substrate, respectively. Dashed color curves are corresponding theoretical results calculated using the structural parameters extracted from the measurements. The dashed gray curves represent the experimental reflectance of bare FP cavity. The inserts are corresponding SEM images for the measured cuboid Au@Ag NRs, the scale bar is $50 \mathrm{~nm}$. e Statistics of decay linewidths for (i) the ELPMs for the NRs on the FP cavity and (ii) bare LPMs for the NRs on the ITO substrate, note that the statistical wavelength range is TM1' $\pm 8, \mathrm{TM}^{\prime} \pm 8$ and TM3' \pm 8 $\mathrm{nm}$, respectively. 
a

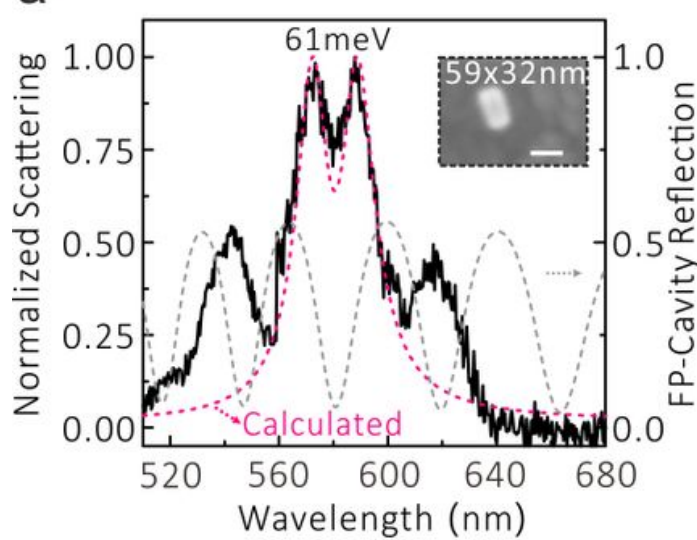

b

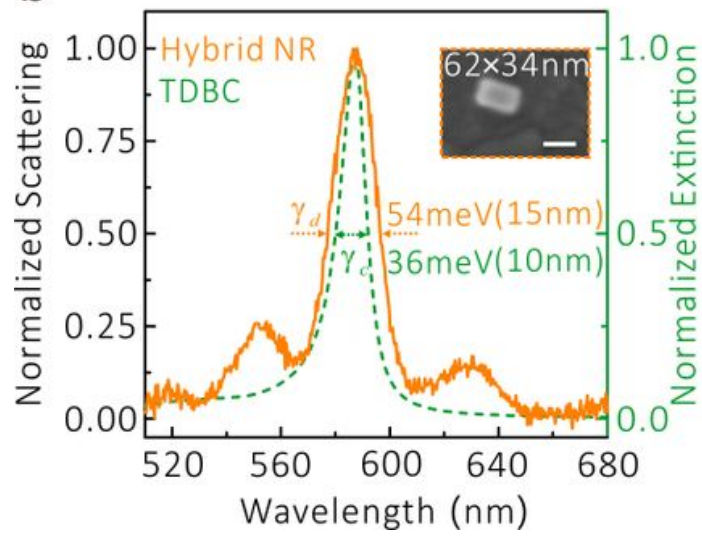

C

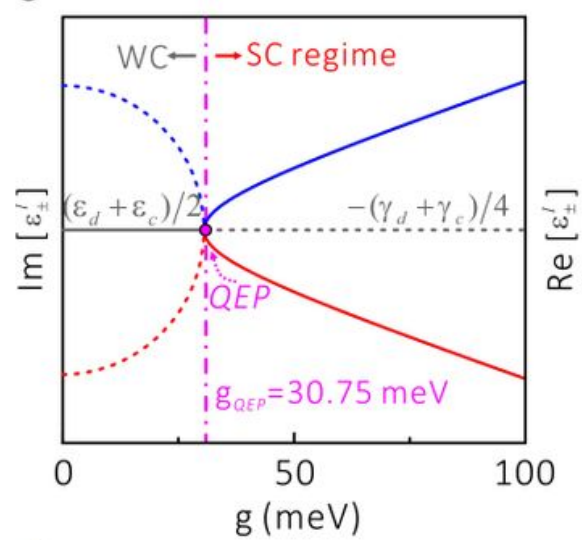

d

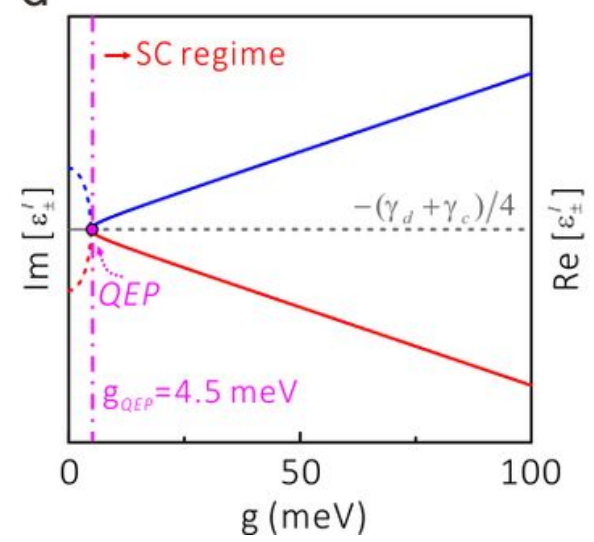

e

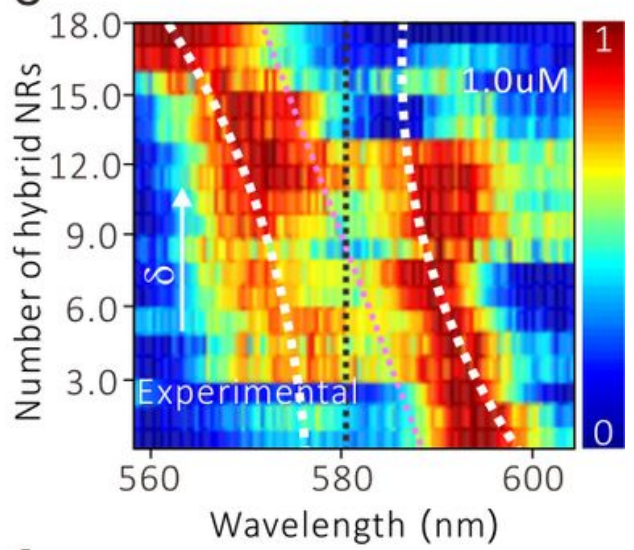

f

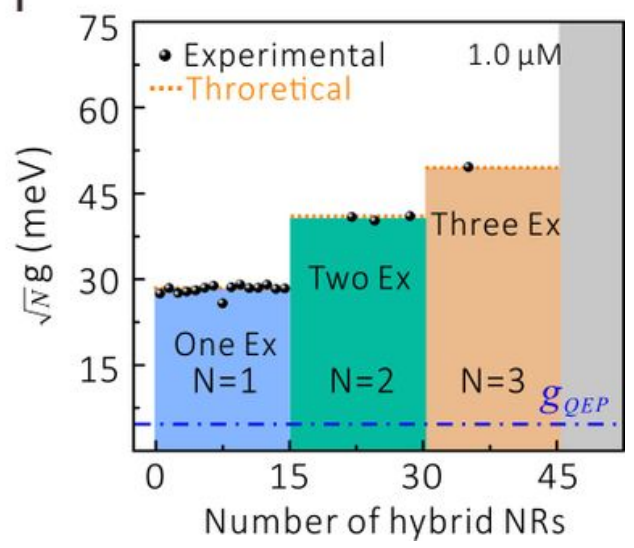

Figure 4

Single-exciton strong coupling in single-exciton-coupled NRs located on the leaky FP cavity. a A strongly coupled Au @ Ag NR/J-aggregate hybrid, measured on FP cavity (Si/SiO2/Si3N4).b Extinction spectra of J-aggregate (dashed green curve) solution and Scattering spectra (orange solid curve) of bare Au @ Ag NR with the size of $62 \times 34 \mathrm{~nm}$ located on FP cavity. c, $d$ Real (solid curve) and imaginary (dashed curve) parts of the eigenenergies in Eq. (1) as a function of $\mathrm{g}$ at resonance $\delta=0$ with (c) $\gamma d=159 \mathrm{meV}, \mathrm{\gamma c}=36$ $\mathrm{meV}$; and (d) $\gamma d=54 \mathrm{meV}, \gamma \mathrm{c}=36 \mathrm{meV}$. WC: Weak coupling; SC: Strong coupling. e Normalized scattering of the individual Au@Ag NR/J-aggregate hybrids isolated from the sample treated 1.0- $\mu \mathrm{M}$ dye solution, and ordered according to detuning. The dashed white lines are theoretical results calculated using Eq. (3) with $\mathrm{N}=1$, yd $\sim 55 \mathrm{meV}$ (Supplementary Fig. 8b), yc $\sim 36 \mathrm{meV}$ and g $\sim 28.8 \mathrm{meV}$. f Quantum steps for the effective coupling coefficient, $\sqrt{ } \mathrm{Ng}$, observed as one, two and three-exciton coupling cases for the hybrid NRs isolated from the sample treated with the $1.0-\mu \mathrm{M}$ dye solution. The scale bar in the insets of (a) and (b) is $50 \mathrm{~nm}$. 
a

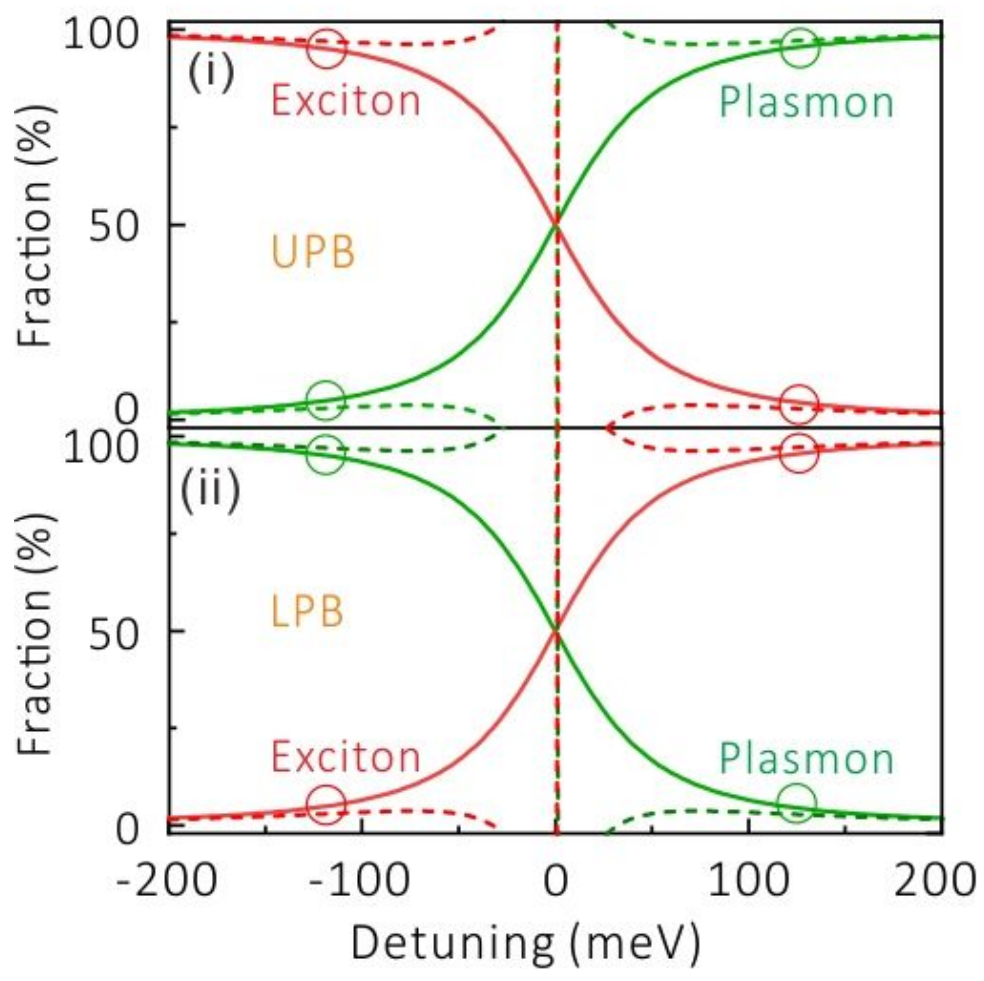

b

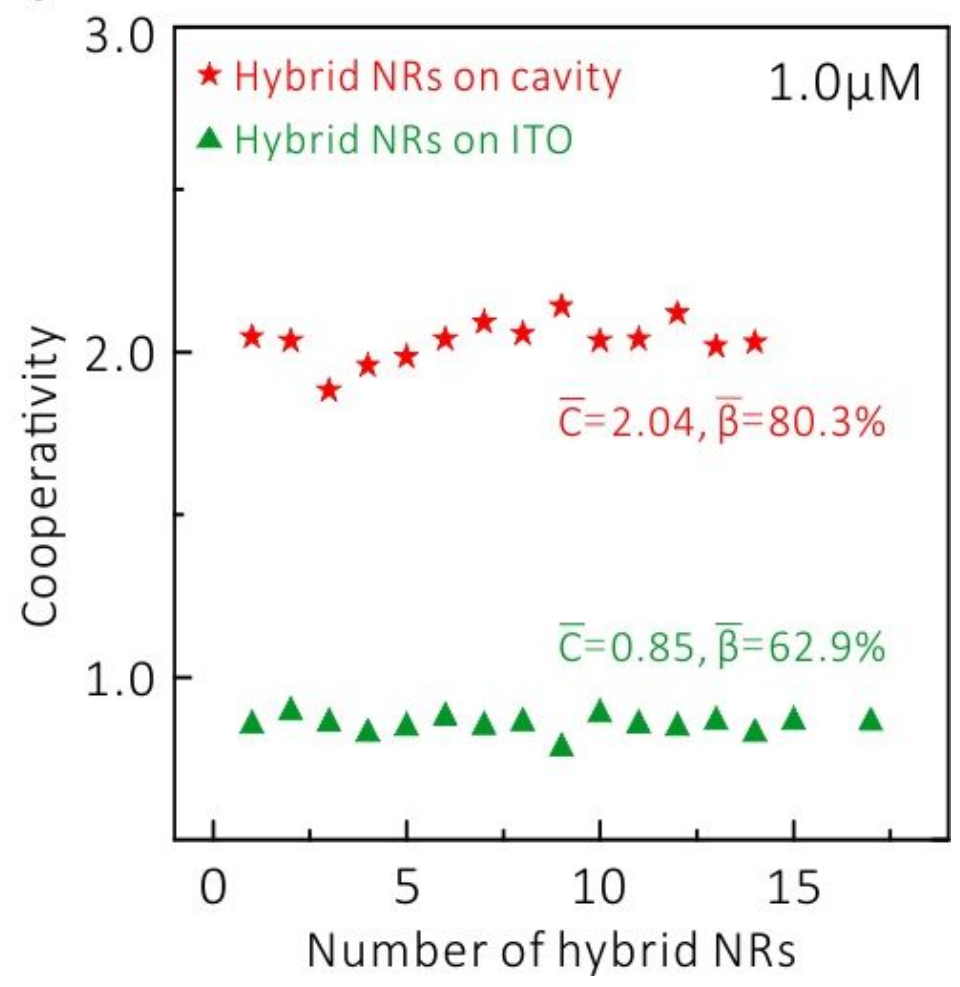

\section{Figure 5}

Influence of controlling gQEP on coherence properties in plasmon-exciton coupling systems. a Plasmonic and exciton fractions for the UPB and LPB of the single-exciton-coupled NRs located on the FP cavity (solid color curves) and the ITO substrate (dashed color curves), In the calculations, the parameters are the same as what have been used in Figs. $1 \mathrm{~d}$ and $4 \mathrm{e}$. $\mathrm{b}$ Cooperativities of the individual single-excitoncoupled NRs located on the FP cavity (red stars) and the ITO substrate (green triangles), which are calculated from the experimental data in Fig. 1c and Fig. 4e, respectively.

\section{Supplementary Files}

This is a list of supplementary files associated with this preprint. Click to download.

- SupplementaryMaterials.doc 